ESAIM: M2AN

Vol. 41, No 3,2007 , pp. 543-574

DOI: $10.1051 / \mathrm{m} 2 \mathrm{an}: 2007026$
ESAIM: Mathematical Modelling and Numerical Analysis

www.edpsciences.org/m2an

\title{
OPTIMAL DESIGN IN SMALL AMPLITUDE HOMOGENIZATION
}

\author{
Grégoire Allaire ${ }^{1}$ And Sergio Gutiérrez ${ }^{1,2}$
}

\begin{abstract}
This paper is concerned with optimal design problems with a special assumption on the coefficients of the state equation. Namely we assume that the variations of these coefficients have a small amplitude. Then, making an asymptotic expansion up to second order with respect to the aspect ratio of the coefficients allows us to greatly simplify the optimal design problem. By using the notion of $H$-measures we are able to prove general existence theorems for small amplitude optimal design and to provide simple and efficient numerical algorithms for their computation. A key feature of this type of problems is that the optimal microstructures are always simple laminates.
\end{abstract}

Mathematics Subject Classification. 15A15, 15A09, 15A23.

Received June 2, 2005. Revised September 6 and December 20, 2006.

\section{INTRODUCTION}

Shape or structural optimization is a very active research topic in applied mathematics, which has seen a burst of new ideas in the last twenty years. A common feature of most of the recently developed methods is to try to circumvent the inceptive ill-posedness of shape optimization problems which manifests itself, in numerical practice, by the occurrence of many local minima, possibly far from being global. Probably the most successful approach is the homogenization method $[1,6,7,19,23]$ : it allows to find a global minimizer in most instances, at the price of introducing composite materials in the optimal shape (a tricky penalization procedure is required for extracting a classical shape out of it). Unfortunately, the rigorous derivation of the homogenized or relaxed formulation of shape optimization is complete only for a few, albeit important, choices of the objective function (mostly self-adjoint problems like compliances or eigenvalues optimization). This difficulty is not just a mathematical problem, but it is also very restrictive from the point of view of numerical applications. Indeed, there are many non-rigorous approaches to treat general objective functions, usually based on some partial relaxations $[3,4,9]$, or ad hoc algorithmic ideas like the SIMP method [6]: none of them is as efficient as the original homogenization method applied to compliance minimization, in the sense that its convergence is neither so smooth, nor so global (the resulting optimum may still depend on the initial guess).

Therefore, many authors have tried to extend the homogenization method to more general objective functions, and in particular to cost functions depending on the gradient of the state (or strain or stress). Although this is a very difficult problem, there has been some results in this direction $[5,11,15,16,22]$. The objective of the present

\footnotetext{
Keywords and phrases. Optimal design, $H$-measures, homogenization.

1 Centre de Mathématiques Appliquées (UMR 7641), École Polytechnique, 91128 Palaiseau, France.

2 Current affiliation: Departamento de Ingeniería Estructural y Geotécnica, Pontificia Universidad Católica de Chile, Santiago Chile, Chile. sgutierr@ing.puc.cl

(c) EDP Sciences, SMAI 2007
} 
paper is also to extend the homogenization method to new objective functions. However, our methodology is quite different: in order to make significant progress, we use a strong simplifying assumption, namely that the two component phases involved in the optimal design have close coefficients or material properties. More precisely we consider two-phase optimal design problems in the context of conductivity or linearized elasticity and we make an asymptotic expansion of the coefficients in terms of the small amplitude parameter that characterizes the variations between the two phases. Restricting ourselves to terms up to second order greatly simplifies the situation. However, the small amplitude optimal design problem is still ill-posed and requires relaxation. The nice feature of our approach is that this relaxation is quite simple because the necessary and delicate tools of homogenization are replaced by more basic results on so-called $H$-measures. These $H$-measures are quadratic default measures, introduced by Gérard [10] and Tartar [21]. They can be interpreted as two-point correlation functions of the underlying microstructure.

We have therefore rigorously derived the relaxed formulation of very general objective functions, including ones depending on the gradient of the state. Furthermore, due to the special "small amplitude" structure of the optimization problem we have devised efficient and simple numerical algorithms for computing the optimal shapes. These algorithms are gradient methods relying on the optimality conditions of the relaxed problem. A key ingredient is that optimal microstructures in small amplitude optimization can always be found in the class of simple or rank-one laminates. In other words, there are only two relevant design parameters in our method: the local volume fraction and the angle of lamination (which governs the anisotropy of the optimal microstructure). Another feature of our small amplitude method is that the coefficient of the state or adjoint equations are uniform and independent of the design. Indeed, all the geometric parameters appear as right hand sides in the equations. This implies a drastic reduction of the CPU cost of the method because, once the rigidity finite element matrix has been factorized by a Cholesky method, it is stored and used throughout the optimization process for different right hand sides at each iteration. We implemented our method only in two space dimensions using the FreeFem ++ package for finite elements [12]. There is no conceptual difficulty in extending the method to three space dimensions where the gain in CPU time is even higher.

Of course, the small amplitude approximation is not really meaningful in the context of "standard" structural optimization which amounts to optimize the distribution of a given material with a very weak one mimicking holes (the so-called ersatz material approach). Indeed, the small amplitude assumption contradicts the fact that the ersatz component is much weaker than the reference one. However, it makes sense, for example, in the context of reinforced plane structures: a typical problem is to find the region where to reinforce the thickness of a plate by pasting some tape on top of it. Our method can be useful for this plane reinforcement problem and our numerical examples can be interpreted in this sense.

The content of the paper is as follows. Section 2 is a brief review of the necessary tools of $H$-measures. Section 3 is devoted to optimal design problems in conductivity, a setting which is simple enough to explain in a clear way our method and give full proofs of our result. Section 4 generalizes the previous one to the linearized elasticity setting. Although the notations are slightly more cumbersome, all the previous results extend easily from conductivity to elasticity. Eventually Section 5 is devoted to algorithmic issues and numerical tests.

\section{A BRIEF REVIEW OF $H$-MEASURES}

The purpose of this section is to recall the necessary results about $H$-measures which were introduced by Gérard [10] and Tartar [21]. It is a default measure which quantifies the lack of compactness of weakly converging sequences in $L^{2}\left(\mathbb{R}^{N}\right)$. More precisely, it indicates where in the physical space, and at which frequency in the Fourier space, are the obstructions to strong convergence. As recognized by Tartar [21], $H$-measures are the right tool for small amplitude homogenization. All results below are due to [10] and [21], to which we refer for complete proofs.

We denote by $\mathbb{S}_{N-1}$ the unit sphere in $\mathbb{R}^{N} . C\left(\mathbb{S}_{N-1}\right)$ is the space of continuous complex-valued functions on $\mathbb{S}_{N-1}$, and $C_{0}\left(\mathbb{R}^{N}\right)$ is that of continuous complex-valued functions decreasing to 0 at infinity in $\mathbb{R}^{N}$. As usual $\bar{z}$ denotes the complex conjugate of the complex number $z$. The Fourier transform operator in $L^{2}\left(\mathbb{R}^{N}\right)$, denoted 
by $\mathcal{F}$, is defined by

$$
(\mathcal{F} \phi)(\xi)=\int_{\mathbb{R}^{N}} \phi(x) \mathrm{e}^{-2 i \pi x \cdot \xi} \mathrm{d} x \quad \forall \phi \in L^{2}\left(\mathbb{R}^{N}\right) .
$$

Theorem 2.1. Let $u_{\epsilon}=\left(u_{\epsilon}^{i}\right)_{1 \leq i \leq p}$ be a sequence of functions defined in $\mathbb{R}^{N}$ with values in $\mathbb{R}^{p}$ which converges weakly to 0 in $L^{2}\left(\mathbb{R}^{N}\right)^{p}$. There exists a subsequence (still denoted by $\epsilon$ ) and a family of complex-valued Radon measures $\left(\mu_{i j}(x, \xi)\right)_{1 \leq i, j \leq p}$ on $\mathbb{R}^{N} \times \mathbb{S}_{N-1}$ such that, for any functions $\phi_{1}(x), \phi_{2}(x) \in C_{0}\left(\mathbb{R}^{N}\right)$ and $\psi(\xi) \in$ $C\left(\mathbb{S}_{N-1}\right)$, it satisfies

$$
\begin{aligned}
& \lim _{\epsilon \rightarrow 0} \int_{\mathbb{R}^{N}} \mathcal{F}\left(\phi_{1} u_{\epsilon}^{i}\right)(\xi) \overline{\mathcal{F}\left(\phi_{2} u_{\epsilon}^{j}\right)}(\xi) \psi\left(\frac{\xi}{|\xi|}\right) \mathrm{d} \xi \\
& =\int_{\mathbb{R}^{N}} \int_{\mathbb{S}_{N-1}} \phi_{1}(x) \bar{\phi}_{2}(x) \psi(\xi) \mu_{i j}(\mathrm{~d} x, \mathrm{~d} \xi) .
\end{aligned}
$$

The matrix of measures $\mu=\left(\mu_{i j}\right)_{1 \leq i, j \leq p}$ is called the $H$-measure of the subsequence $u_{\epsilon}$. It is hermitian and non-negative, i.e.

$$
\mu_{i j}=\bar{\mu}_{j i}, \sum_{i, j=1}^{p} \lambda_{i} \bar{\lambda}_{j} \mu_{i j} \geq 0 \forall \lambda \in \mathbb{C}^{p} .
$$

If we consider a sequence $u_{\epsilon}$ which converges weakly in $L^{2}\left(\mathbb{R}^{N}\right)^{p}$ to a limit $u$ (instead of 0 ), then, applying Theorem 2.1 to $\left(u_{\epsilon}-u\right)$, and taking $\psi \equiv 1$, we obtain a representation formula for the limit of quadratic forms of $u_{\epsilon}$

$$
\lim _{\epsilon \rightarrow 0} \int_{\mathbb{R}^{N}} \phi_{1} \overline{\phi_{2}} u_{\epsilon}^{i} u_{\epsilon}^{j} \mathrm{~d} x=\int_{\mathbb{R}^{N}} \phi_{1} \overline{\phi_{2}} u^{i} u^{j} \mathrm{~d} x+\int_{\mathbb{R}^{N}} \int_{\mathbb{S}_{N-1}} \phi_{1}(x) \bar{\phi}_{2}(x) \mu_{i j}(\mathrm{~d} x, \mathrm{~d} \xi) .
$$

Therefore the $H$-measure appears as a default measure which gives a precise representation of the compactness default, taking into account the directions of the oscillation.

One of the main interest of Theorem 2.1 is its generalization to a broader class of quadratic forms of $u_{\epsilon}$ in the context of pseudo-differential operators (see Sect. 18.1 in [13]). Recall that a standard pseudo-differential operator $q$ is defined through its symbol $\left(q_{i j}(x, \xi)\right)_{1 \leq i, j \leq p} \in C^{\infty}\left(\mathbb{R}^{N} \times \mathbb{R}^{N}\right)$ by

$$
(q u)_{i}(x)=\sum_{j=1}^{p} \mathcal{F}^{-1}\left(q_{i j}(x, \cdot) \mathcal{F} u_{j}(\cdot)\right)(x)
$$

for any smooth and compactly supported function $u$. We consider only so-called polyhomogeneous pseudodifferential operators of order 0 , i.e. whose symbol $\left(q_{i j}(x, \xi)\right)_{1 \leq i, j \leq p}$ is homogeneous of degree 0 in $\xi$ and with compact support in $x$. Recall that such polyhomogeneous pseudo-differential operators of order 0 are bounded operators in $L^{2}\left(\mathbb{R}^{N}\right)^{p}$.

Theorem 2.2. Let $u_{\epsilon}$ be a sequence which converges weakly to 0 in $L^{2}\left(\mathbb{R}^{N}\right)^{p}$. There exist a subsequence and an $H$-measure $\mu$ such that, for any polyhomogeneous pseudo-differential operator $q$ of degree 0 with symbol $\left(q_{i j}(x, \xi)\right)_{1 \leq i, j \leq p}$,

$$
\lim _{\epsilon \rightarrow 0} \int_{\mathbb{R}^{N}} q\left(u_{\epsilon}\right) \cdot \bar{u}_{\epsilon} \mathrm{d} x=\int_{\mathbb{R}^{N}} \int_{\mathbb{S}_{N-1}} \sum_{i, j=1}^{p} q_{i j}(x, \xi) \mu_{i j}(\mathrm{~d} x, \mathrm{~d} \xi) .
$$

We now recall the particular case of characteristic functions [14,21].

Lemma 2.3. Let $\chi_{\epsilon}(x)$ be a sequence of characteristic functions that weakly-* converges to a limit $\theta(x)$ in $L^{\infty}(\Omega ;[0,1])$. Then the corresponding $H$-measure $\mu$ for the sequence $\left(\chi_{\epsilon}-\theta\right)$ is necessarily of the type

$$
\mu(\mathrm{d} x, \mathrm{~d} \xi)=\theta(x)(1-\theta(x)) \nu(\mathrm{d} x, \mathrm{~d} \xi)
$$


where, for given $x$, the measure $\nu(\mathrm{d} x, \mathrm{~d} \xi)$ is a probability measure with respect to $\xi$, i.e. $\nu \in \mathcal{P}\left(\Omega, \mathbb{S}_{N-1}\right)$ with

$$
\mathcal{P}\left(\Omega, \mathbb{S}_{N-1}\right)=\left\{\begin{array}{l}
\nu(x, \xi) \text { Radon measure on } \Omega \times \mathbb{S}_{N-1} \text { such that: } \\
\nu \geq 0, \int_{\mathbb{S}_{N-1}} \nu(x, \xi) \mathrm{d} \xi=1 \text { a.e. } x \in \Omega
\end{array}\right\} .
$$

Conversely, for any such probability measure $\nu \in \mathcal{P}\left(\Omega, \mathbb{S}_{N-1}\right)$ there exists a sequence $\chi_{\epsilon}$, which weakly-* converges to $\theta$ in $L^{\infty}(\Omega ;[0,1])$, such that $\theta(1-\theta) \nu$ is the $H$-measure of $\left(\chi_{\epsilon}-\theta\right)$.

Remark 2.4. In the periodic setting the notion of $H$-measure has a very simple interpretation and it is often called two-point correlation function in the context of composite materials [18]. Indeed, let $u(x, y)$ be a smooth function defined on $\Omega \times Y$, with $Y=(0,1)^{N}$, such that $y \rightarrow u(x, y)$ is $Y$-periodic. Assuming that $\int_{Y} u(x, y) \mathrm{d} y=0$, it is easily seen that $u_{\epsilon}(x)=u(x, x / \epsilon)$ converges weakly to 0 in $L^{2}(\Omega)$. By using the Fourier series decomposition in $Y$, the $H$-measure $\mu$ of $u_{\epsilon}$ is simple to compute. Introducing

$$
u(x, y)=\sum_{k \in \mathbb{Z}^{N}} \hat{u}(x, k) \mathrm{e}^{2 i \pi k \cdot y}
$$

we deduce

where $\delta$ is the Dirac mass.

$$
\mu=\sum_{k \neq 0 \in \mathbb{Z}^{N}}|\hat{u}(x, k)|^{2} \delta\left(\xi-\frac{k}{|k|}\right)
$$

\section{A MODEL PROBlem in CONDUCTIVITY}

\subsection{Small amplitude asymptotic}

Let us consider mixtures of two conducting phases characterized by two symmetric positive definite tensors $A^{0}$ and $A^{1}$. We denote by $\eta$ the amplitude or contrast or aspect ratio between the two materials. In other words, we assume that

$$
A^{1}=A^{0}(1+\eta)
$$

The range of $\eta$ is restricted to $(-1 ;+\infty)$, but in the sequel we shall assume that $\eta$ is a small parameter, i.e. $|\eta| \ll 1$. Denoting by $\chi$ the characteristic function of the region occupied by phase $A^{1}$, we define a conductivity tensor

$$
A(x)=(1-\chi(x)) A^{0}+\chi(x) A^{1}=A^{0}(1+\eta \chi(x)) .
$$

For a smooth bounded open set $\Omega \subset \mathbb{R}^{N}$, with boundary $\partial \Omega=\Gamma_{D} \cup \Gamma_{N}$, and for given source terms $f \in L^{2}(\Omega)$ and $g \in L^{2}(\partial \Omega)$, we consider the following boundary value problem

$$
\left.\begin{array}{r}
-\operatorname{div}(A \nabla u)=f \text { in } \Omega \\
u=0 \text { on } \Gamma_{D} \\
A \nabla u \cdot n=g \text { on } \Gamma_{N},
\end{array}\right\}
$$

which admits a unique solution in $H^{1}(\Omega)$. Typically we want to minimize an objective function of the type

$$
J(\chi)=\int_{\Omega} j_{1}(u) \mathrm{d} x+\int_{\Gamma_{N}} j_{2}(u) \mathrm{d} s
$$

where the boundary integral is defined only on $\Gamma_{N}$ since $u=0$ is fixed on $\Gamma_{D}$. We assume that the integrands $j_{i}$ are of class $C^{3}$ with adequate growth conditions. For example, we assume that there exists a constant $C>0$ such that, for any $u \in \mathbb{R}$,

$$
\left|j_{i}(u)\right| \leq C\left(|u|^{2}+1\right), \quad\left|j_{i}^{\prime}(u)\right| \leq C(|u|+1), \quad\left|j_{i}^{\prime \prime}(u)\right| \leq C .
$$


Of course, more subtle and less restrictive assumptions are possible.

Assuming that the two phases have prescribed volume fractions, $\Theta$ for $A^{1}$ and $1-\Theta$ for $A^{0}$, with $\Theta \in(0,1)$, we define a set of admissible designs

$$
\mathcal{U}_{a d}=\left\{\chi \in L^{\infty}(\Omega ;\{0,1\}), \text { such that } \int_{\Omega} \chi(x) \mathrm{d} x=\Theta|\Omega|\right\} .
$$

We are ready to define the starting point of our study.

Definition 3.1. We call "large amplitude" optimal design problem the following optimization problem

$$
\inf _{\chi \in \mathcal{U}_{a d}} J(\chi)
$$

Although problem (5) has been extensively studied by means of homogenization theory $[1,7,19,23]$, we propose yet another approach based on the simplifying assumption that the amplitude parameter $\eta$ is small. The small amplitude asymptotic of (5) will then be relaxed by using $H$-measures theory. Our motivation for introducing this new method is that many simple generalizations of (5) can not be solved by the homogenization method whereas they are amenable to our small amplitude approach. Examples of this are problems in which the objective function depends on the state gradient $\nabla u$ or upon the strain or stress tensor in the case of elasticity. For the sake of pedagogy we begin with the simple example (5), for which we already know the complete relaxation and thus a comparison between the large and small amplitude cases is possible. The novelty of our approach will come afterwards when studying the gradient case in Section 3.5 for which there is no complete treatment of the large amplitude case. The examples in elasticity are also new and will be treated in Section 4.

Assuming that the amplitude or contrast $\eta$ is small, we perform a second-order expansion in the state equation and in the objective function. Since the coefficient matrix $A$ in (3) is an affine function of $\eta$, the solution $u \in H^{1}(\Omega)$ is analytic with respect to $\eta$, and we can write

$$
u=u^{0}+\eta u^{1}+\eta^{2} u^{2}+O\left(\eta^{3}\right) .
$$

Plugging this ansatz in (3) yields three equations for $\left(u^{0}, u^{1}, u^{2}\right)$

$$
\left.\begin{array}{c}
-\operatorname{div}\left(A^{0} \nabla u^{0}\right)=f, \\
u^{0}=0 \text { on } \Gamma_{D} \\
A^{0} \nabla u^{0} \cdot n=g \text { on } \Gamma_{N},
\end{array}\right\}
$$

Remark that $u^{0}$ does not depend on $\chi$ and thus only $u^{1}, u^{2}$ depends on $\chi$. Similarly, we make a Taylor expansion in the objective function to get

$$
\begin{aligned}
J(\chi)=\int_{\Omega} j_{1}\left(u^{0}\right) \mathrm{d} x+\eta & \int_{\Omega} j_{1}^{\prime}\left(u^{0}\right) u^{1} \mathrm{~d} x+\eta^{2} \int_{\Omega}\left(j_{1}^{\prime}\left(u^{0}\right) u^{2}+\frac{1}{2} j_{1}^{\prime \prime}\left(u^{0}\right)\left(u^{1}\right)^{2}\right) \mathrm{d} x \\
& +\int_{\Gamma_{N}} j_{2}\left(u^{0}\right) \mathrm{d} s+\eta \int_{\Gamma_{N}} j_{2}^{\prime}\left(u^{0}\right) u^{1} \mathrm{~d} s+\eta^{2} \int_{\Gamma_{N}}\left(j_{2}^{\prime}\left(u^{0}\right) u^{2}+\frac{1}{2} j_{2}^{\prime \prime}\left(u^{0}\right)\left(u^{1}\right)^{2}\right) \mathrm{d} s+O\left(\eta^{3}\right) .
\end{aligned}
$$


Neglecting the remainder term we introduce a function $\mathcal{J}_{s a}$ which only depends on $u^{0}, u^{1}, u^{2}$

$$
\begin{aligned}
\mathcal{J}_{s a}\left(u^{0}, u^{1}, u^{2}\right)= & \int_{\Omega} j_{1}\left(u^{0}\right) \mathrm{d} x+\eta \int_{\Omega} j_{1}^{\prime}\left(u^{0}\right) u^{1} \mathrm{~d} x \\
& +\eta^{2} \int_{\Omega}\left(j_{1}^{\prime}\left(u^{0}\right) u^{2}+\frac{1}{2} j_{1}^{\prime \prime}\left(u^{0}\right)\left(u^{1}\right)^{2}\right) \mathrm{d} x \\
& +\int_{\Gamma_{N}} j_{2}\left(u^{0}\right) \mathrm{d} s+\eta \int_{\Gamma_{N}} j_{2}^{\prime}\left(u^{0}\right) u^{1} \mathrm{~d} s \\
& +\eta^{2} \int_{\Gamma_{N}}\left(j_{2}^{\prime}\left(u^{0}\right) u^{2}+\frac{1}{2} j_{2}^{\prime \prime}\left(u^{0}\right)\left(u^{1}\right)^{2}\right) \mathrm{d} s .
\end{aligned}
$$

Finally, we can define the typical shape optimization problem we are interested in.

Definition 3.2. We call "small amplitude" optimal design problem the second-order asymptotic of problem (5), namely

$$
\inf _{\chi \in \mathcal{U}_{a d}}\left\{J_{s a}(\chi)=\mathcal{J}_{s a}\left(u^{0}, u^{1}, u^{2}\right)\right\}
$$

where $\mathcal{J}_{s a}$ is defined by (10) and $u^{0}, u^{1}, u^{2}$ are solutions of the state equations (7-9) respectively.

The rest of the paper is devoted to the study of the shape optimization problem (11) and its various generalizations.

Remark 3.3. The asymptotic expansion (6) is actually uniform with respect to the characteristic function (a fact that we shall use later in Rem. 3.11). Indeed, introducing

$$
r_{\eta}=\eta^{-3}\left(u-u^{0}-\eta u^{1}-\eta^{2} u^{2}\right),
$$

it is easily seen to satisfy

$$
\left.\begin{array}{r}
-\operatorname{div}\left(A \nabla r_{\eta}\right)=\operatorname{div}\left(\chi A^{0} \nabla u^{2}\right) \\
r_{\eta}=0 \\
\text { on } \Gamma_{D} \\
A \nabla r_{\eta} \cdot n=-\chi A^{0} \nabla u^{2} \cdot n \quad \text { on } \Gamma_{N},
\end{array}\right\}
$$

from which we deduce the a priori estimate

$$
\left\|\nabla r_{\eta}\right\|_{L^{2}(\Omega)^{N}} \leq \frac{C}{\min (1,1+\eta)}
$$

where the constant $C>0$ does not depend on $\eta$ nor on $\chi$.

\subsection{Relaxation by $H$-measures}

As most optimal design problems, the small amplitude problem (11) is ill-posed in the sense that it does not admit a minimizer in general. Therefore we relax it by using $H$-measures.

Remark 3.4. For technical reasons when using $H$-measures, we need to assume that $\nabla u^{0}$ is continuous inside $\Omega$. Since $u^{0}$ is a solution to (7) which has constant coefficients and $f$ as its right hand side, this regularity property is easily deduced if $f$ is smooth enough, say $f \in L^{\infty}(\Omega)$. From now on we shall make this technical assumption.

The general procedure for computing the relaxation of (11) is to consider a sequence (minimizing or not) of characteristic functions $\chi_{n}$ and to pass to the limit in the objective function (11) and its associated state equations. Up to a subsequence there exists a limit density $\theta$ such that $\chi_{n}$ converges weakly-* to $\theta$ in $L^{\infty}(\Omega ;[0,1])$. From now on we restrict our attention to this converging subsequence. We denote by $u^{0}, u_{n}^{1}, u_{n}^{2}$ the solutions 
of (7), (8), and (9) respectively, associated to $\chi_{n}$ (recall that (7) does not depend on $\chi_{n}$ ). In a first step, it is easy to pass to the limit in the variational formulation of (8) to obtain that $u_{n}^{1}$ converges weakly to $u^{1}$ in $H^{1}(\Omega)$ which is the solution of

$$
\left.\begin{array}{rl}
-\operatorname{div}\left(A^{0} \nabla u^{1}\right) & =\operatorname{div}\left(\theta A^{0} \nabla u^{0}\right) \text { in } \Omega \\
u^{1} & =0 \text { on } \Gamma_{D} \\
A^{0} \nabla u^{1} \cdot n & =-\theta A^{0} \nabla u^{0} \cdot n \text { on } \Gamma_{N} .
\end{array}\right\} .
$$

The main difficulty comes from (9) where we need to pass to the limit in the product $\chi_{n} \nabla u_{n}^{1}$ : since this term is quadratic, we can use $H$-measure theory. We know from Lemma 2.3 that, up to another subsequence, $\chi_{n}$ admits an $H$-measure of the type

$$
\mu(\mathrm{d} x, \mathrm{~d} \xi)=\theta(x)(1-\theta(x)) \nu(\mathrm{d} x, \mathrm{~d} \xi)
$$

where $\nu$ is a probability measure with respect to $\xi$. Restricting again our attention to this subsequence, we can now state the following result.

Lemma 3.5. The sequence $u_{n}^{2}$ converges weakly in $H^{1}(\Omega)$ to a limit $u^{2}$ which is the unique solution of

$$
\left.\begin{array}{rl}
-\operatorname{div}\left(A^{0} \nabla u^{2}\right) & =\operatorname{div}\left(\theta A^{0} \nabla u^{1}\right)-\operatorname{div}\left(\theta(1-\theta) A^{0} M A^{0} \nabla u^{0}\right) \text { in } \Omega, \\
u^{2} & =0 \text { on } \Gamma_{D}, \\
A^{0} \nabla u^{2} \cdot n & =-\theta A^{0} \nabla u^{1} \cdot n+\theta(1-\theta) A^{0} M A^{0} \nabla u^{0} \cdot n \text { on } \Gamma_{N},
\end{array}\right\}
$$

where the matrix $M(x)$ is defined by

$$
M=\int_{\mathbb{S}^{N-1}} \frac{\xi \otimes \xi}{A^{0} \xi \cdot \xi} \nu(x, \mathrm{~d} \xi)
$$

We postpone for a moment the proof of Lemma 3.5 and we go on in the relaxation process by passing to the limit in the objective function $J_{s a}\left(\chi_{n}\right)$. Since the embeddings of $H^{1}(\Omega)$ in $L^{2}(\Omega)$ and in $L^{2}\left(\Gamma_{N}\right)$ are compact, we easily obtain

$$
\lim _{n \rightarrow+\infty} J_{s a}\left(\chi_{n}\right)=J_{s a}^{*}(\theta, \nu)=\mathcal{J}_{s a}\left(u^{0}, u^{1}, u^{2}\right)
$$

where $u^{0}, u^{1}, u^{2}$ are now solutions of the relaxed state equations (7), (12), (13), respectively. Remark that in the present case the expression of the objective function is the same before and after relaxation. It is now a standard matter to deduce the following result.

Proposition 3.6. The relaxation of (11) is thus

$$
\min _{(\theta, \nu) \in \mathcal{U}_{a d}^{*}}\left\{J_{s a}^{*}(\theta, \nu)=\mathcal{J}_{s a}\left(u^{0}, u^{1}, u^{2}\right)\right\}
$$

where $\mathcal{J}_{\text {sa }}\left(u^{0}, u^{1}, u^{2}\right)$ is defined by $(10), u^{0}, u^{1}, u^{2}$ are solutions of (7), (12), (13), respectively, and $\mathcal{U}_{\text {ad }}^{*}$ is defined by

$$
\mathcal{U}_{a d}^{*}=\left\{(\theta, \nu) \in L^{\infty}(\Omega ;[0,1]) \times \mathcal{P}\left(\Omega, \mathbb{S}_{N-1}\right) \text { such that } \int_{\Omega} \theta(x) \mathrm{d} x=\Theta|\Omega|\right\},
$$

where the set of probability measures $\mathcal{P}\left(\Omega, \mathbb{S}_{N-1}\right)$ is defined in (2). More precisely,

1. there exists at least one minimizer $(\theta, \nu)$ of $(15)$;

2. any minimizer $(\theta, \nu)$ of $(15)$ is attained by a minimizing sequence $\chi_{n}$ of (11) in the sense that $\chi_{n}$ converges weakly-* to $\theta$ in $L^{\infty}(\Omega), \nu$ is the $H$-measure of $\left(\chi_{n}-\theta\right)$, and $\lim _{n \rightarrow+\infty} J_{s a}\left(\chi_{n}\right)=J_{s a}^{*}(\theta, \nu)$;

3. any minimizing sequence $\chi_{n}$ of (11) converges in the previous sense to a minimizer $(\theta, \nu)$ of $(15)$.

Proof of Lemma 3.5. The variational formulation of (9) is

$$
\int_{\Omega} A^{0} \nabla u_{n}^{2} \cdot \nabla \phi \mathrm{d} x=-\int_{\Omega} \chi_{n} A^{0} \nabla u_{n}^{1} \cdot \nabla \phi \mathrm{d} x
$$


for any test function $\phi \in H^{1}(\Omega)$ which vanishes on $\Gamma_{D}$. The sequence $u_{n}^{2}$ is obviously bounded in $H^{1}(\Omega)$ and, up to a subsequence, it converges weakly to a limit $u^{2}$ in $H^{1}(\Omega)$. To obtain the limit equation satisfied by $u^{2}$, we need to pass to the limit in the right hand side of (17). Assume for the moment that $\Omega=\mathbb{R}^{N}$. Then, using our assumption that $\nabla u^{0}$ is continuous and following Tartar (see Sect. 4.2 in [21]) or Gérard (see Thm. 1 in [10]), we deduce from (8) that $\nabla u_{n}^{1}$ depends linearly on $\chi_{n}$ through a pseudo-differential operator $q$, homogeneous of order 0 , the symbol of which is

$$
q(x, \xi)=-\frac{A^{0} \nabla u^{0}(x) \cdot \xi}{A^{0} \xi \cdot \xi} \xi .
$$

Although $q(x, \xi)$ is merely continuous in $x$, the result of Thm. 2.2 still holds true (see Sect. 4.2 in [21] or Thm. 2 in $[10])$ and we deduce that

$$
\begin{aligned}
\lim _{n \rightarrow+\infty} \int_{\Omega} \chi_{n} A^{0} \nabla u_{n}^{1} \cdot \nabla \phi \mathrm{d} x & =\int_{\Omega} \theta A^{0} \nabla u^{1} \cdot \nabla \phi \mathrm{d} x \\
& -\int_{\Omega} \theta(1-\theta) A^{0} M A^{0} \nabla u^{0} \cdot \nabla \phi \mathrm{d} x
\end{aligned}
$$

where the $H$-measure moment matrix $M(x)$ is defined by (14). Consequently the limit of (17) is

$$
\int_{\Omega} A^{0} \nabla u^{2} \cdot \nabla \phi \mathrm{d} x=-\int_{\Omega} \theta A^{0} \nabla u^{1} \cdot \nabla \phi \mathrm{d} x+\int_{\Omega} \theta(1-\theta) A^{0} M A^{0} \nabla u^{0} \cdot \nabla \phi \mathrm{d} x
$$

which is precisely the variational formulation of (13). In the case of a bounded domain $\Omega$ we need a further localization argument that goes as follows. Let $\left(\zeta_{k}\right)_{k \geq 1}$ be a sequence of smooth compactly supported functions in $C_{c}^{\infty}(\Omega)$ which converges strongly to 1 in $L^{2}(\Omega)$. We rewrite the right hand side of (17) as

$$
I_{n}=-\int_{\Omega} \zeta_{k} \chi_{n} A^{0} \nabla u_{n}^{1} \cdot \nabla \phi \mathrm{d} x+\int_{\Omega}\left(\zeta_{k}-1\right) \chi_{n} A^{0} \nabla u_{n}^{1} \cdot \nabla \phi \mathrm{d} x
$$

For a smooth test function $\phi$ we can bound the last term in (19) by

$$
\left\|\zeta_{k}-1\right\|_{L^{2}(\Omega)}\left\|\nabla u_{n}^{1}\right\|_{L^{2}(\Omega)}\|\nabla \phi\|_{L^{\infty}(\Omega)} \leq C\left\|\zeta_{k}-1\right\|_{L^{2}(\Omega)}
$$

because $u_{n}^{1}$ is bounded in $H^{1}(\Omega)$. Therefore, the last term of (19) converges to 0 as $k$ goes to infinity, uniformly with respect to $n$. Let $\psi_{k}$ be another smooth compactly supported function in $C_{c}^{\infty}(\Omega)$ such that $\psi_{k} \equiv 1$ inside the support of $\zeta_{k}$. The first term on the right hand side of (19) is thus equal to

$$
-\int_{\Omega} \zeta_{k}\left(\psi_{k} \chi_{n}\right) A^{0} \nabla\left(\psi_{k} u_{n}^{1}\right) \cdot \nabla \phi \mathrm{d} x
$$

A simple computation shows that $\left(\psi_{k} u_{n}^{1}\right)=\tilde{u}_{n}^{1}+\check{u}_{n}^{1}$ on the support of $\zeta_{k}$, where the last two functions are the solutions of the following equations in the whole space $\mathbb{R}^{N}$

$$
\begin{aligned}
& -\operatorname{div}\left(A^{0} \nabla \tilde{u}_{n}^{1}\right)=\operatorname{div}\left(\left(\psi_{k} \chi_{n}\right) A^{0} \nabla u^{0}\right) \quad \text { in } \mathbb{R}^{N}, \\
& -\operatorname{div}\left(A^{0} \nabla \check{u}_{n}^{1}\right)=-\operatorname{div}\left(u_{n}^{1} A^{0} \nabla \psi_{k}\right)-A^{0} \nabla \psi_{k} \cdot\left(\chi_{n} \nabla u^{0}+\nabla u_{n}^{1}\right) \quad \text { in } \mathbb{R}^{N} .
\end{aligned}
$$

Clearly the sequence $\check{u}_{n}^{1}$ converges strongly in $H^{1}\left(\mathbb{R}^{N}\right)$ to a limit $\check{u}^{1}$, while $\tilde{u}_{n}^{1}$ converges merely weakly in $H^{1}\left(\mathbb{R}^{N}\right)$ to a limit $\tilde{u}^{1}$ which satisfy $u^{1}=\check{u}^{1}+\tilde{u}^{1}$ on the support of $\zeta_{k}$. However, as in the case $\Omega=\mathbb{R}^{N}, \nabla \tilde{u}_{n}^{1}$ depends linearly on $\left(\psi_{k} \chi_{n}\right)$ through the pseudo-differential operator $q$ of symbol (18). We can therefore apply Theorem 2 of [10] to the product of $\chi_{n}$ and $\nabla \tilde{u}_{n}^{1}$. Eventually, the limit of the first term on the right hand side 
of (19) becomes

$$
\begin{aligned}
& \lim _{n \rightarrow+\infty} \int_{\Omega} \zeta_{k}\left(\psi_{k} \chi_{n}\right) A^{0} \nabla\left(\check{u}_{n}^{1}+\tilde{u}_{n}^{1}\right) \cdot \nabla \phi \mathrm{d} x=\int_{\Omega} \zeta_{k}\left(\psi_{k} \theta\right) A^{0} \nabla \check{u}^{1} \cdot \nabla \phi \mathrm{d} x \\
&+\lim _{n \rightarrow+\infty} \int_{\Omega} \zeta_{k}\left(\psi_{k} \chi_{n}\right) A^{0} \nabla \tilde{u}_{n}^{1} \cdot \nabla \phi \mathrm{d} x \\
&=\int_{\Omega} \zeta_{k} \theta A^{0} \nabla u^{1} \cdot \nabla \phi \mathrm{d} x-\int_{\Omega} \zeta_{k} \theta(1-\theta) A^{0} M A^{0} \nabla u^{0} \cdot \nabla \phi \mathrm{d} x,
\end{aligned}
$$

and passing to the last limit $k \rightarrow+\infty$ we obtain the desired result.

Remark 3.7. A simpler, albeit formal, method for computing the limits of $u_{n}^{1}$ and $u_{n}^{2}$ is to assume that the sequence $\chi_{n}$ of characteristic functions is periodically oscillating, i.e. $\chi_{n}(x)=\chi(x, n x)$ where $y \rightarrow \chi(x, y)$ is $Y$-periodic. Then, using formal two-scale asymptotic expansions it is possible to compute the limits of $u_{n}^{1}$ and $u_{n}^{2}$, as well as the first-order corrector term for $u_{n}^{1}$, i.e.

$$
u_{n}^{1}(x)=u^{1}(x)+\frac{1}{n} u^{11}(x, n x)+O\left(\frac{1}{n^{2}}\right) .
$$

Making a Fourier expansion of $\chi$ as

$$
\chi(x, y)=\sum_{k \in \mathbb{Z}^{N}} \hat{\chi}(x, k) \mathrm{e}^{2 i \pi k \cdot y}
$$

we can compute explicitly

$$
u^{11}(x, y)=\sum_{k \neq 0 \in \mathbb{Z}^{N}} i \hat{\chi}(x, k) \frac{A^{0} \nabla u^{0} \cdot k}{2 \pi A^{0} k \cdot k} \mathrm{e}^{2 i \pi k \cdot y} .
$$

This allows to recover the limit problem for $u^{2}$ with the $H$-measure being given, as in Remark 2.4, by

$$
\nu(x, \xi)=\frac{1}{\theta(1-\theta)} \sum_{k \neq 0 \in \mathbb{Z}^{N}}|\hat{\chi}(x, k)|^{2} \delta\left(\xi-\frac{k}{|k|}\right) .
$$

Remark 3.8. In this section, as well as in the previous one, the integrand of the objective function was not directly depending on the characteristic function $\chi$ (but implicitly through the solutions of the state equations). Actually our approach does not apply directly to an objective function where the integrand depends on $\chi$ as, for example,

$$
J(\chi)=\int_{\Omega}\left((1-\chi) j_{0}(u)+\chi j_{1}(u)\right) \mathrm{d} x .
$$

Indeed, after the second order expansion in the amplitude parameter $\eta$ we get

$$
\mathcal{J}_{s a}\left(u^{0}, u^{1}, u^{2}\right)=\int_{\Omega} j\left(u^{0}\right) \mathrm{d} x+\eta \int_{\Omega} j^{\prime}\left(u^{0}\right) u^{1} \mathrm{~d} x+\eta^{2} \int_{\Omega}\left(j^{\prime}\left(u^{0}\right) u^{2}+\frac{1}{2} j^{\prime \prime}\left(u^{0}\right)\left(u^{1}\right)^{2}\right) \mathrm{d} x
$$

with $j=(1-\chi) j_{0}+\chi j_{1}$. It is clear that the last term in (20) is cubic with respect to $\chi$, which means that we can not use $H$-measures (which are merely quadratic in $\chi$ ) to pass to the limit and relax such an objective function $\mathcal{J}_{s a}$. However, if we assume that the two integrands have also a small contrast of order $\eta$, i.e.

$$
j_{1}(v)=j_{0}(v)+\eta k(v) \quad \forall v \in \mathbb{R}
$$


then, the second order expansion yields

$$
\begin{aligned}
\mathcal{J}_{s a}\left(u^{0}, u^{1}, u^{2}\right)= & \int_{\Omega} j_{0}\left(u^{0}\right) \mathrm{d} x+\eta \int_{\Omega}\left(j_{0}^{\prime}\left(u^{0}\right) u^{1}+\chi k\left(u_{0}\right)\right) \mathrm{d} x \\
& +\eta^{2} \int_{\Omega}\left(j^{\prime}\left(u^{0}\right) u^{2}+\frac{1}{2} j^{\prime \prime}\left(u^{0}\right)\left(u^{1}\right)^{2}+\chi k^{\prime}\left(u_{0}\right) u_{1}\right) \mathrm{d} x
\end{aligned}
$$

in which there are only at most quadratic terms in $\chi$. We can thus pass to the limit by using $H$-measures as before and obtain a relaxation result.

\subsection{Relaxation before small amplitude asymptotic}

In this section we proceed in reverse order compared to the previous one. Namely, we first relax the large amplitude problem (5) by using homogenization theory and then we make a small amplitude asymptotic. The resulting small-amplitude relaxed problem turns out to be the same as (15) (which is not surprising as explained below in Rem. 3.11).

The relaxation of (5) is a classical result [19] that we now recall. Let us first define the so-called $G$-closure set $G_{\theta} \subset \mathbb{R}^{N^{2}}$ of all homogenized tensors obtained by mixing the two phases $A^{0}$ and $A^{1}$ in proportions $1-\theta$ and $\theta$ respectively. If the phases are isotropic this set $G_{\theta}$ of symmetric matrices has an explicit characterization in terms of the matrix eigenvalues (see e.g. $[1,7,18,19,23]$ ) which is not required in the sequel.

Proposition 3.9. The relaxed formulation of (5) is

$$
\min _{\left(\theta, A^{\text {eff }}\right) \in \mathcal{U}_{a d}^{\text {eff }}}\left\{J^{*}\left(\theta, A^{\text {eff }}\right)=\int_{\Omega} j_{1}(u) \mathrm{d} x+\int_{\Gamma_{N}} j_{2}(u) \mathrm{d} s\right\}
$$

where

$$
\mathcal{U}_{a d}^{\text {eff }}=\left\{\begin{array}{c}
\left(\theta, A^{\text {eff }}\right) \in L^{\infty}\left(\Omega ;[0,1] \times \mathbb{R}^{\left.N^{2}\right), \text { such that: }}\right. \\
\int_{\Omega} \theta(x) \mathrm{d} x=\Theta|\Omega|, A^{\mathrm{eff}}(x) \in G_{\theta(x)} \text { a.e. } x \in \Omega
\end{array}\right\},
$$

and $u$ is the unique solution in $H^{1}(\Omega)$ of the homogenized problem

$$
\left.\begin{array}{r}
-\operatorname{div}\left(A^{\mathrm{eff}} \nabla u\right)=f \text { in } \Omega \\
u=0 \\
A^{\mathrm{eff}} \nabla u \cdot n=g \text { on } \Gamma_{D} \\
\text { on } \Gamma_{N} .
\end{array}\right\}
$$

More precisely,

1. there exists at least one minimizer $\left(\theta, A^{\mathrm{eff}}\right)$ of (21);

2. any minimizer $\left(\theta, A^{\mathrm{eff}}\right)$ of (21) is attained by a minimizing sequence $\chi_{n}$ of (5) in the sense that $\chi_{n}$ converges weakly-* to $\theta$ in $L^{\infty}(\Omega), A^{n}=\chi_{n} A^{1}+\left(1-\chi_{n}\right) A^{0} H$-converges to $A^{\mathrm{eff}}$, and $\lim _{n \rightarrow+\infty} J\left(\chi_{n}\right)=$ $J^{*}\left(\theta, A^{\mathrm{eff}}\right)$;

3. any minimizing sequence $\chi_{n}$ of (5) converges in the previous sense to a minimizer $\left(\theta, A^{\mathrm{eff}}\right)$ of $(21)$.

The main result of this section is that the small amplitude asymptotic of (21) is precisely (15).

Proposition 3.10. The shape optimization problem (15) is the second-order approximation of (21) for small amplitude $\eta$.

Proof. For $|\eta| \ll 1$, we use the small amplitude formula devised by Tartar [21] for the homogenized tensor $A^{\text {eff }}$

$$
A^{\mathrm{eff}}=A^{0}+\theta \eta A^{0}-\theta(1-\theta) \eta^{2} A^{0}\left(\int_{S^{N-1}} \frac{\xi \otimes \xi}{A^{0} \xi \cdot \xi} \mathrm{d} \nu\right) A^{0}+O\left(\eta^{3}\right)
$$


where $\nu$ is the $H$-measure induced by the sequence $\left\{\chi_{n}\right\}$ such that $A^{n}=\chi_{n} A^{1}+\left(1-\chi_{n}\right) A^{0} H$-converges to $A^{\mathrm{eff}}$ (remark that such a formula was already obtained in the mechanical literature by formal arguments, for example in the case of periodic composites). Therefore, up to second-order in $\eta$, the homogenized tensor $A^{\text {eff }}$ is completely characterized by the density $\theta$ and the $H$-measure $\nu$. We can thus replace $\mathcal{U}_{a d}^{\text {eff }}$ by $\mathcal{U}_{a d}^{*}$. On the other hand, writing the solution of (23) as

$$
u=u^{0}+\eta u^{1}+\eta^{2} u^{2}+O\left(\eta^{3}\right),
$$

it is easily seen that $u^{0}, u^{1}, u^{2}$ are precisely the solutions of $(7),(12),(13)$, respectively. Finally, we make a second order asymptotic expansion of $J^{*}\left(\theta, A^{\text {eff }}\right)$ to precisely obtain $J_{s a}^{*}(\theta, \nu)$.

Remark 3.11. Proposition 3.10 is not surprising because we know from Remark 3.3 that the second-order expansion of the state $u$ with respect to $\eta$ is uniform in $\chi$. So, if we add the following assumption about the remainder of the Taylor expansion of the cost functions $j_{i}$

$$
\left|j_{i}(u)-j_{i}\left(u^{0}\right)-j_{i}^{\prime}\left(u^{0}\right)\left(u-u^{0}\right)-\frac{1}{2} j_{i}^{\prime \prime}\left(u^{0}\right)\left(u-u^{0}\right)^{2}\right| \leq C\left|u-u^{0}\right|^{2} o(1),
$$

where $o(1)$ is a uniformly bounded function which goes to 0 with $\left|u-u^{0}\right|$, then the small amplitude approximation (11) of the original large amplitude shape optimization problem (5) is uniform with respect to the characteristic function $\chi$. In particular, it guarantees that the minimizer of the relaxed small amplitude problem (15) is close, up to third order in $\eta$, to minimizing sequences of (5). This result confirms the interest of the small amplitude approximation which is easier to relax.

\subsection{Optimality conditions}

The goal of this section is to simplify the relaxed small amplitude optimization problem (15) by using information coming from its optimality conditions. The main result is that optimal microstructures for (15) can always be found in the class of simple laminates (i.e. rank-one laminates).

Proposition 3.12. The relaxed small amplitude problem (15) can be equivalently solved by restricting the set of probability measures $\mathcal{P}\left(\Omega, \mathbb{S}_{N-1}\right)$, defined by (2), to its subset of Dirac masses. More precisely, there exists an optimal design solution of

$$
\min _{(\theta, \nu) \in \mathcal{U}_{a d}^{s l}} J_{s a}^{*}(\theta, \nu)
$$

where $\mathcal{U}_{a d}^{s l} \subset \mathcal{U}_{\text {ad }}^{*}$ is defined by

$$
\mathcal{U}_{a d}^{s l}=\left\{\begin{array}{l}
(\theta, \nu) \in L^{\infty}(\Omega ;[0,1]) \times \mathcal{P}\left(\Omega, \mathbb{S}_{N-1}\right), \text { such that: } \\
\int_{\Omega} \theta(x) \mathrm{d} x=\Theta|\Omega|, \nu(x, \xi)=\delta\left(\xi-\xi^{*}(x)\right) \text { a.e. } x \in \Omega
\end{array}\right\} .
$$

Furthermore, the optimal Dirac mass $H$-measure in (25) does not depend on the density $\theta$.

Remark 3.13. The main consequence of Proposition 3.12 is that not all possible composite materials have to be considered in the relaxed small amplitude problem (15) but just the simple laminates of rank one. This is, of course, a drastic simplification which is reminiscent of a similar one in the "large amplitude" case due to Raitums [20]. However, we shall see that it is much more general since it holds true for all generalizations of (15) investigated in this paper. Another interesting consequence of Proposition 3.12 is that the optimization with respect to $\nu$ can be done once and for all at the beginning of the optimization process since it is independent of the exact values of $\theta$. 
Proof. To simplify the formula for $J_{s a}^{*}(\theta, \nu)$ which is implicit in $\nu$, we introduce an adjoint state $p^{0}$ solution in $H^{1}(\Omega)$ of

$$
\left.\begin{array}{rl}
-\operatorname{div}\left(A^{0} \nabla p^{0}\right) & =j_{1}^{\prime}\left(u^{0}\right) \text { in } \Omega \\
p^{0} & =0 \text { on } \Gamma_{D} \\
A^{0} \nabla p^{0} \cdot n & =j_{2}^{\prime}\left(u^{0}\right) \text { on } \Gamma_{N} .
\end{array}\right\}
$$

Remark that, like $u^{0}$, the adjoint state $p^{0}$ does not depend on $(\theta, \nu)$. The goal of this adjoint state is to eliminate $u^{2}$ in $J_{s a}^{*}(\theta, \nu)$. Indeed multiplying (27) by $u^{2}$ and integrating by parts, and doing the same for (13) multiplied by $p^{0}$, we obtain

$$
\begin{gathered}
\int_{\Omega} j_{1}^{\prime}\left(u^{0}\right) u^{2} \mathrm{~d} x+\int_{\Gamma_{N}} j_{2}^{\prime}\left(u^{0}\right) u^{2} \mathrm{~d} s=-\int_{\Omega} \theta A^{0} \nabla u^{1} \cdot \nabla p^{0} \mathrm{~d} x \\
+\int_{\Omega} \theta(1-\theta) A^{0} M A^{0} \nabla u^{0} \cdot \nabla p^{0} \mathrm{~d} x,
\end{gathered}
$$

which is now explicitly affine in $M$, defined by (14), and thus in $\nu$. More precisely we have

$$
\begin{aligned}
J_{s a}^{*}(\theta, \nu)= & \int_{\Omega} j_{1}\left(u^{0}\right) \mathrm{d} x+\eta \int_{\Omega} j_{1}^{\prime}\left(u^{0}\right) u^{1} \mathrm{~d} x+\eta^{2} \int_{\Omega} \frac{1}{2} j_{1}^{\prime \prime}\left(u^{0}\right)\left(u^{1}\right)^{2} \mathrm{~d} x \\
& +\int_{\Gamma_{N}} j_{2}\left(u^{0}\right) \mathrm{d} s+\eta \int_{\Gamma_{N}} j_{2}^{\prime}\left(u^{0}\right) u^{1} \mathrm{~d} s+\eta^{2} \int_{\Gamma_{N}} \frac{1}{2} j_{2}^{\prime \prime}\left(u^{0}\right)\left(u^{1}\right)^{2} \mathrm{~d} s \\
& -\eta^{2} \int_{\Omega} \theta A^{0} \nabla u^{1} \cdot \nabla p^{0} \mathrm{~d} x+\eta^{2} \int_{\Omega} \theta(1-\theta) A^{0} M A^{0} \nabla u^{0} \cdot \nabla p^{0} \mathrm{~d} x .
\end{aligned}
$$

Only the last term in (28) depend (linearly) on $\nu$ since $u^{0}$ and $p^{0}$ are independent of $\nu$ (and $\theta$ ). Minimizing $J_{s a}^{*}(\theta, \nu)$ with respect to $\nu$ amounts to minimize a scalar affine function on the convex set of probability measures $\mathcal{P}\left(\Omega, \mathbb{S}_{N-1}\right)$. Therefore any minimizer $\nu^{*}$ can be replaced by another minimizer which is a Dirac mass concentrated at a direction $\xi^{*}$ which minimizes the function

$$
\frac{\xi \otimes \xi}{A^{0} \xi \cdot \xi} A^{0} \nabla u^{0} \cdot A^{0} \nabla p^{0}
$$

Remark that $\xi^{*}$ does not depend on $\theta$. Furthermore, replacing a minimizer $\nu^{*}$ by the Dirac mass concentrated at $\xi^{*}$ does not change $\theta, u^{0}, u^{1}$ and $p^{0}$. Thus one can restrict the minimization in $\nu$ to the subset of $\mathcal{P}\left(\Omega, \mathbb{S}_{N-1}\right)$ made of Dirac masses of the type $\nu(x, \xi)=\delta\left(\xi-\xi^{0}(x)\right)$.

Remark 3.14. In the case where $A^{0}$ is isotropic, we can compute explicitly the optimal direction $\xi^{*}$. If either $\nabla u^{0}$ or $\nabla p^{0}$ vanishes, then any direction is optimal. Otherwise, the optimal direction is easily seen to be

$$
\xi^{*}=\frac{e-e^{\prime}}{\left\|e-e^{\prime}\right\|} \text { if } e \neq e^{\prime}, \quad \xi^{*} \perp e \text { if } e=e^{\prime},
$$

where $e=\nabla u^{0} /\left|\nabla u^{0}\right|$ and $e^{\prime}=\nabla p^{0} /\left|\nabla p^{0}\right|$. 
After elimination of the measure $\nu$, i.e. incorporating the optimal Dirac mass concentrated on $\xi^{*}(x)$, we obtain an objective function that depends only on $\theta$

$$
\begin{aligned}
J_{s a}^{*}(\theta)= & \int_{\Omega} j_{1}\left(u^{0}\right) \mathrm{d} x+\int_{\Gamma_{N}} j_{2}\left(u^{0}\right) \mathrm{d} s+\eta \int_{\Omega} j_{1}^{\prime}\left(u^{0}\right) u^{1} \mathrm{~d} x+\eta \int_{\Gamma_{N}} j_{2}^{\prime}\left(u^{0}\right) u^{1} \mathrm{~d} s \\
& +\frac{1}{2} \eta^{2} \int_{\Omega} j_{1}^{\prime \prime}\left(u^{0}\right)\left(u^{1}\right)^{2} \mathrm{~d} x+\frac{1}{2} \eta^{2} \int_{\Gamma_{N}} j_{2}^{\prime \prime}\left(u^{0}\right)\left(u^{1}\right)^{2} \mathrm{~d} s \\
& -\eta^{2} \int_{\Omega} \theta A^{0} \nabla u^{1} \cdot \nabla p^{0} \mathrm{~d} x+\eta^{2} \int_{\Omega} \theta(1-\theta) A^{0} M^{*} A^{0} \nabla u^{0} \cdot \nabla p^{0} \mathrm{~d} x
\end{aligned}
$$

with

$$
M^{*}=\frac{\xi^{*} \otimes \xi^{*}}{A^{0} \xi^{*} \cdot \xi^{*}}
$$

We can also eliminate $u^{1}$ in the first order term in $\eta$. Once again we use the adjoint $p^{0}$ : multiplying (27) by $u^{1}$ and integrating by parts, and doing the same for (12) multiplied by $p^{0}$, we obtain

$$
\int_{\Omega} j_{1}^{\prime}\left(u^{0}\right) u^{1} \mathrm{~d} x+\int_{\Gamma_{N}} j_{2}^{\prime}\left(u^{0}\right) u^{1} \mathrm{~d} s=-\int_{\Omega} \theta A^{0} \nabla u^{0} \cdot \nabla p^{0} \mathrm{~d} x
$$

Therefore we deduce

$$
\begin{aligned}
J_{s a}^{*}(\theta)= & \int_{\Omega} j_{1}\left(u^{0}\right) \mathrm{d} x+\int_{\Gamma_{N}} j_{2}\left(u^{0}\right) \mathrm{d} s-\eta \int_{\Omega} \theta A^{0} \nabla u^{0} \cdot \nabla p^{0} \mathrm{~d} x \\
& +\frac{1}{2} \eta^{2} \int_{\Omega} j_{1}^{\prime \prime}\left(u^{0}\right)\left(u^{1}\right)^{2} \mathrm{~d} x+\frac{1}{2} \eta^{2} \int_{\Gamma_{N}} j_{2}^{\prime \prime}\left(u^{0}\right)\left(u^{1}\right)^{2} \mathrm{~d} s \\
& -\eta^{2} \int_{\Omega} \theta A^{0} \nabla u^{1} \cdot \nabla p^{0} \mathrm{~d} x+\eta^{2} \int_{\Omega} \theta(1-\theta) A^{0} M^{*} A^{0} \nabla u^{0} \cdot \nabla p^{0} \mathrm{~d} x
\end{aligned}
$$

It is then a simple matter to compute the derivative of $J_{s a}^{*}$ with respect to $\theta$.

Lemma 3.15. The objective function $J_{s a}^{*}(\theta)$ is Fréchet differentiable and its derivative in the direction $s \in$ $L^{\infty}(\Omega)$ is given by

$$
\begin{aligned}
\frac{\partial J_{s a}^{*}}{\partial \theta}(s)= & -\eta \int_{\Omega} s A^{0} \nabla u^{0} \cdot \nabla p^{0} \mathrm{~d} x-\eta^{2} \int_{\Omega} s A^{0} \nabla u^{1} \cdot \nabla p^{0} \mathrm{~d} x \\
& -\eta^{2} \int_{\Omega} s A^{0} \nabla u^{0} \cdot \nabla p^{1} \mathrm{~d} x+\eta^{2} \int_{\Omega} s(1-2 \theta) A^{0} M^{*} A^{0} \nabla u^{0} \cdot \nabla p^{0} \mathrm{~d} x,
\end{aligned}
$$

where $p^{1}$ is another adjoint state, defined as the solution in $H^{1}(\Omega)$ of

$$
\left.\begin{array}{rll}
-\operatorname{div}\left(A^{0} \nabla p^{1}\right) & =j_{1}^{\prime \prime}\left(u^{0}\right) u^{1}+\operatorname{div}\left(\theta A^{0} \nabla p^{0}\right) & \text { in } \Omega \\
p^{1} & =0 \text { on } \Gamma_{D} \\
A^{0} \nabla p^{1} \cdot n & =j_{2}^{\prime \prime}\left(u^{0}\right) u^{1}-\theta A^{0} \nabla p^{0} \cdot n & \text { on } \Gamma_{N} .
\end{array}\right\}
$$

Proof. To compute the derivative of $J_{s a}^{*}$ with respect to $\theta$, we first define the derivative of $u^{1}$ with respect to $\theta$ in the direction $s \in L^{\infty}(\Omega)$, denoted by $z=\frac{\partial u^{1}}{\partial \theta}(s)$. It is easily seen that $u^{1} \in H^{1}(\Omega)$ is Fréchet differentiable 
with respect to $\theta$ and that

$$
\left.\begin{array}{rll}
-\operatorname{div}\left(A^{0} \nabla z\right) & =\operatorname{div}\left(s A^{0} \nabla u^{0}\right) & \text { in } \Omega \\
z & =0 \text { on } \Gamma_{D} \\
A^{0} \nabla z \cdot n & =-s A^{0} \nabla u^{0} \cdot n \text { on } \Gamma_{N} .
\end{array}\right\}
$$

Then we have

$$
\begin{aligned}
\frac{\partial J_{s a}^{*}}{\partial \theta}(s)= & -\eta \int_{\Omega} s A^{0} \nabla u^{0} \cdot \nabla p^{0} \mathrm{~d} x+\eta^{2} \int_{\Omega} j_{1}^{\prime \prime}\left(u^{0}\right) u^{1} z \mathrm{~d} x \\
& +\eta^{2} \int_{\Gamma_{N}} j_{2}^{\prime \prime}\left(u^{0}\right) u^{1} z \mathrm{~d} s-\eta^{2} \int_{\Omega} s A^{0} \nabla u^{1} \cdot \nabla p^{0} \mathrm{~d} x \\
& -\eta^{2} \int_{\Omega} \theta A^{0} \nabla z \cdot \nabla p^{0} \mathrm{~d} x+\eta^{2} \int_{\Omega} s(1-2 \theta) A^{0} M^{*} A^{0} \nabla u^{0} \cdot \nabla p^{0} \mathrm{~d} x .
\end{aligned}
$$

To eliminate $z$ in the above expression, we use the adjoint state $p^{1}$. Multiplying (29) by $z$ and (30) by $p^{1}$ we get

$$
\int_{\Omega} j_{1}^{\prime \prime}\left(u^{0}\right) u^{1} z \mathrm{~d} x-\int_{\Omega} \theta A^{0} \nabla p^{0} \cdot \nabla z \mathrm{~d} x+\int_{\Gamma_{N}} j_{2}^{\prime \prime}\left(u^{0}\right) u^{1} z \mathrm{~d} s=-\int_{\Omega} s A^{0} \nabla u^{0} \cdot \nabla p^{1} \mathrm{~d} x
$$

which yields the desired formula.

Remark 3.16. In the case of compliance minimization we have that $j_{1}(u)=f u$ and $j_{2}=g u$. Thus, we deduce that the first adjoint is $p^{0}=u^{0}$. Then, an optimal direction $\xi^{*}$ is a vector orthogonal to $A^{0} \nabla u^{0}$, which implies that $M^{*} A^{0} \nabla u^{0}=0$. In other words, we obtain

$$
J_{s a}^{*}(\theta)=\int_{\Omega} f u^{0} \mathrm{~d} x+\int_{\Gamma_{N}} g u^{0} \mathrm{~d} s-\eta \int_{\Omega} \theta A^{0} \nabla u^{0} \cdot \nabla u^{0} \mathrm{~d} x-\eta^{2} \int_{\Omega} \theta A^{0} \nabla u^{1} \cdot \nabla u^{0} \mathrm{~d} x .
$$

By using equation (12) we can rewrite the second order term in $\eta$ such that

$$
J_{s a}^{*}(\theta)=\int_{\Omega} f u^{0} \mathrm{~d} x+\int_{\Gamma_{N}} g u^{0} \mathrm{~d} s-\eta \int_{\Omega} \theta A^{0} \nabla u^{0} \cdot \nabla u^{0} \mathrm{~d} x+\eta^{2} \int_{\Omega} A^{0} \nabla u^{1} \cdot \nabla u^{1} \mathrm{~d} x .
$$

Since $u^{1}$ depends linearly on $\theta$ we obtain that $J_{s a}^{*}(\theta)$ is a non-negative quadratic function of $\theta$ and thus is convex. In particular, any local minimizer is necessarily a global minimizer.

\subsection{Gradient based objective function}

We now study an objective function that depends on the gradient

$$
J(\chi)=\int_{\Omega} j(\nabla u) \mathrm{d} x
$$

where $j: \mathbb{R}^{N} \rightarrow \mathbb{R}$ is a $C^{3}$ function satisfying adequate growth conditions. For example, a simple growth condition is that there exists a constant $C>0$ such that, for any $\lambda \in \mathbb{R}^{N}$,

$$
|j(\lambda)| \leq C\left(|\lambda|^{2}+1\right), \quad\left|j^{\prime}(\lambda)\right| \leq C(|\lambda|+1), \quad\left|j^{\prime \prime}(\lambda)\right| \leq C .
$$


The function $J$ is associated to the state equation (3) for $u$. Making a small amplitude second-order expansion in $J$ we obtain the objective function associated to the state equations (7), (8), and (9)

$$
\begin{aligned}
J_{s a}(\chi)= & \int_{\Omega} j\left(\nabla u^{0}\right) \mathrm{d} x+\eta \int_{\Omega} j^{\prime}\left(\nabla u^{0}\right) \cdot \nabla u^{1} \mathrm{~d} x \\
& +\eta^{2} \int_{\Omega}\left(j^{\prime}\left(\nabla u^{0}\right) \cdot \nabla u^{2}+\frac{1}{2} j^{\prime \prime}\left(\nabla u^{0}\right) \nabla u^{1} \cdot \nabla u^{1}\right) \mathrm{d} x .
\end{aligned}
$$

The small amplitude problem we consider is thus

$$
\inf _{\chi \in \mathcal{U}_{a d}} J_{s a}(\chi)
$$

where $J_{s a}$ is defined by $(31)$ and $u^{0}, u^{1}, u^{2}$ are solutions of the state equations $(7),(8),(9)$ respectively.

We now compute the relaxation of (32). Taking a sequence of characteristic functions $\chi_{n}$ which converges weakly-* to $\theta$ in $L^{\infty}(\Omega ;[0,1])$, we already know that its associated solutions $u_{n}^{1}, u_{n}^{2}$ converge weakly to $u^{1}, u^{2}$ in $H^{1}(\Omega)$ which are the solutions of $(12),(13)$, respectively. However, to compute the limit of $J_{s a}\left(\chi_{n}\right)$ we need to pass to the limit in the quadratic function $j^{\prime \prime}\left(\nabla u^{0}\right) \nabla u_{n}^{1} \cdot \nabla u_{n}^{1}$.

As already said in Section 3.2, we know that $\nabla u_{n}^{1}$ depends linearly on $\chi_{n}$ through a pseudo-differential operator $q$, which, in the case $\Omega=\mathbb{R}^{N}$, is homogeneous of order 0 with symbol

$$
q(x, \xi)=-\frac{A^{0} \nabla u^{0}(x) \cdot \xi}{A^{0} \xi \cdot \xi} \xi .
$$

If $\Omega$ is not equal to the whole space, we need a localization argument as in the proof of Lemma 3.5. Although the present localization argument is slightly more complex (in particular it uses the fact that $\nabla u_{n}^{1}$ is uniformly bounded in $L^{p}(\Omega)^{N}$ for some $p>2$ ), its spirit is exactly the same and we do not give any details for the sake of brevity. Therefore, applying a result in the spirit of Theorem 2.2 (revisited by Lem. 3.5), we deduce that

$$
\begin{aligned}
\lim _{n \rightarrow+\infty} \int_{\Omega} j^{\prime \prime}\left(\nabla u^{0}\right) \nabla u_{n}^{1} \cdot \nabla u_{n}^{1} \mathrm{~d} x & =\int_{\Omega} j^{\prime \prime}\left(\nabla u^{0}\right) \nabla u^{1} \cdot \nabla u^{1} \mathrm{~d} x \\
& +\int_{\Omega} \int_{\mathbb{S}_{N-1}} \theta(1-\theta)\left(\frac{A^{0} \nabla u^{0} \cdot \xi}{A^{0} \xi \cdot \xi}\right)^{2} j^{\prime \prime}\left(\nabla u^{0}\right) \xi \cdot \xi \nu(\mathrm{d} x, \mathrm{~d} \xi) .
\end{aligned}
$$

Introducing a matrix $N(x)$ defined by

$$
N=\frac{1}{2} \int_{\mathbb{S}^{N-1}} \frac{j^{\prime \prime}\left(\nabla u^{0}\right) \xi \cdot \xi}{\left(A^{0} \xi \cdot \xi\right)^{2}} \xi \otimes \xi \nu(\mathrm{d} \xi)
$$

we finally obtain the relaxed objective function

$$
\begin{aligned}
J_{s a}^{*}(\theta, \nu)= & \int_{\Omega} j\left(\nabla u^{0}\right) \mathrm{d} x+\eta \int_{\Omega} j^{\prime}\left(\nabla u^{0}\right) \cdot \nabla u^{1} \mathrm{~d} x \\
& +\eta^{2} \int_{\Omega}\left(j^{\prime}\left(\nabla u^{0}\right) \cdot \nabla u^{2}+\frac{1}{2} j^{\prime \prime}\left(\nabla u^{0}\right) \nabla u^{1} \cdot \nabla u^{1}\right) \mathrm{d} x \\
& +\eta^{2} \int_{\Omega} \theta(1-\theta) A^{0} N A^{0} \nabla u^{0} \cdot \nabla u^{0} \mathrm{~d} x,
\end{aligned}
$$

where $u^{0}, u^{1}, u^{2}$ are solutions of the relaxed state equations (7), (12), (13), respectively. We have thus proved the following result. 
Proposition 3.17. The relaxation of (32) is

$$
\min _{(\theta, \nu) \in \mathcal{U}_{a d}^{*}} J_{s a}^{*}(\theta, \nu)
$$

where $\mathcal{U}_{\text {ad }}^{*}$ is defined by (16). More precisely,

1. there exists at least one minimizer $(\theta, \nu)$ of $(35)$;

2. any minimizer $(\theta, \nu)$ of (35) is attained by a minimizing sequence $\chi_{n}$ of (32) in the sense that $\chi_{n}$ converges weakly-* to $\theta$ in $L^{\infty}(\Omega), \nu$ is the $H$-measure of $\left(\chi_{n}-\theta\right)$, and $\lim _{n \rightarrow+\infty} J_{s a}\left(\chi_{n}\right)=J_{s a}^{*}(\theta, \nu)$;

3. any minimizing sequence $\chi_{n}$ of (32) converges in the previous sense to a minimizer $(\theta, \nu)$ of $(35)$.

Remark 3.18. Proposition 3.17 gives a complete relaxation of the small amplitude problem (32) while there is no such result for the corresponding large amplitude problem (except in a few special cases; see [5,22]).

As in the previous section (see Prop. 3.12) we can simplify (35) by considering only simple laminates instead of all possible composites.

Proposition 3.19. The relaxed small amplitude problem (35) can be equivalently solved by restricting the set of probability measures $\mathcal{P}\left(\Omega, \mathbb{S}_{N-1}\right)$, defined by (2), to its subset of Dirac masses. More precisely, there exists an optimal design solution of

$$
\min _{(\theta, \nu) \in \mathcal{U}_{a d}^{s l}} J_{s a}^{*}(\theta, \nu)
$$

where $\mathcal{U}_{a d}^{s l} \subset \mathcal{U}_{a d}^{*}$ is defined by (26). Furthermore, the optimal Dirac mass H-measure in (36) does not depend on the density $\theta$.

Proof. To eliminate $u^{2}$ in the previous formula for $J_{s a}^{*}$ we introduce a first adjoint state $p^{0}$ as the solution in $H^{1}(\Omega)$ of

We thus obtain

$$
\left.\begin{array}{rll}
-\operatorname{div}\left(A^{0} \nabla p^{0}\right) & =-\operatorname{div} j^{\prime}\left(\nabla u^{0}\right) & \text { in } \Omega \\
p^{0} & =0 \text { on } \Gamma_{D} & \\
A^{0} \nabla p^{0} \cdot n & =j^{\prime}\left(\nabla u^{0}\right) \cdot n & \text { on } \Gamma_{N} .
\end{array}\right\}
$$

$$
\int_{\Omega} j^{\prime}\left(\nabla u^{0}\right) \cdot \nabla u^{2} \mathrm{~d} x=-\int_{\Omega} \theta A^{0} \nabla u^{1} \cdot \nabla p^{0} \mathrm{~d} x+\int_{\Omega} \theta(1-\theta) A^{0} M A^{0} \nabla u^{0} \cdot \nabla p^{0} \mathrm{~d} x .
$$

The objective function is thus linear with respect to the $H$-measure $\nu$

$$
\begin{aligned}
J_{s a}^{*}(\theta, \nu)= & \int_{\Omega} j\left(\nabla u^{0}\right) \mathrm{d} x+\eta \int_{\Omega} j^{\prime}\left(\nabla u^{0}\right) \cdot \nabla u^{1} \mathrm{~d} x-\eta^{2} \int_{\Omega} \theta A^{0} \nabla u^{1} \cdot \nabla p^{0} \mathrm{~d} x \\
& +\frac{\eta^{2}}{2} \int_{\Omega} j^{\prime \prime}\left(\nabla u^{0}\right) \nabla u^{1} \cdot \nabla u^{1} \mathrm{~d} x \\
& +\eta^{2} \int_{\Omega} \theta(1-\theta)\left(A^{0} N A^{0} \nabla u^{0} \cdot \nabla u^{0}+A^{0} M A^{0} \nabla u^{0} \cdot \nabla p^{0}\right) \mathrm{d} x .
\end{aligned}
$$

To minimize $J_{s a}^{*}$ which is linear in $\nu$ is it enough to take $\nu$ as a Dirac mass in a direction $\xi^{*}$ which minimizes in $\mathbb{S}^{N-1}$ the following function of $\xi$

$$
h(\xi)=\frac{\left(A^{0} \nabla u^{0} \cdot \xi\right)\left(A^{0} \nabla p^{0} \cdot \xi\right)}{A^{0} \xi \cdot \xi}+\frac{1}{2} \frac{\left(j^{\prime \prime}\left(\nabla u^{0}\right) \xi \cdot \xi\right)\left(A^{0} \nabla u^{0} \cdot \xi\right)^{2}}{\left(A^{0} \xi \cdot \xi\right)^{2}} .
$$

Remark that the optimal direction $\xi^{*}(x)$ depends on $\nabla u^{0}(x)$ and $\nabla p^{0}(x)$ but not on $\theta(x)$. 
After choosing $\nu$ as the optimal Dirac mass concentrated at $\xi^{*}(x)$, we obtain an objective function that depends only on $\theta$. Using again the adjoint $p^{0}$ we obtain a new formula for the objective function

$$
\begin{aligned}
J_{s a}^{*}(\theta) & =\int_{\Omega} j\left(\nabla u^{0}\right) \mathrm{d} x-\eta \int_{\Omega} \theta A^{0} \nabla u^{0} \cdot \nabla p^{0} \mathrm{~d} x-\eta^{2} \int_{\Omega} \theta A^{0} \nabla u^{1} \cdot \nabla p^{0} \mathrm{~d} x \\
& +\frac{\eta^{2}}{2} \int_{\Omega} j^{\prime \prime}\left(\nabla u^{0}\right) \nabla u^{1} \cdot \nabla u^{1} \mathrm{~d} x+\eta^{2} \int_{\Omega} \theta(1-\theta) h\left(\xi^{*}\right) \mathrm{d} x .
\end{aligned}
$$

To differentiate $J_{s a}^{*}$ with respect to $\theta$ we already know that $u^{1}$ is Fréchet differentiable with respect to $\theta$, and thus it remains simply to eliminate the derivative of $u^{1}$. Therefore we define a second adjoint state $p^{1}$ as the solution in $H^{1}(\Omega)$ of

$$
\left.\begin{array}{rl}
-\operatorname{div}\left(A^{0} \nabla p^{1}\right) & =\operatorname{div}\left(j^{\prime \prime}\left(\nabla u^{0}\right) \nabla u^{1}\right)-\operatorname{div}\left(\theta A^{0} \nabla p^{0}\right) \text { in } \Omega \\
p^{1} & =0 \text { on } \Gamma_{D} \\
A^{0} \nabla p^{1} \cdot n & =\left(\theta A^{0} \nabla p^{0}-j^{\prime \prime}\left(\nabla u^{0}\right) \nabla u^{1}\right) \cdot n \text { on } \Gamma_{N} .
\end{array}\right\}
$$

We deduce that

$$
\begin{aligned}
\frac{\partial J_{s a}^{*}}{\partial \theta}(s)= & -\eta \int_{\Omega} s A^{0} \nabla u^{0} \cdot \nabla p^{0} \mathrm{~d} x+\eta^{2} \int_{\Omega} s A^{0} \nabla u^{0} \cdot \nabla p^{1} \mathrm{~d} x \\
& -\eta^{2} \int_{\Omega} s A^{0} \nabla u^{1} \cdot \nabla p^{0} \mathrm{~d} x+\eta^{2} \int_{\Omega} s(1-2 \theta) h\left(\xi^{*}\right) \mathrm{d} x
\end{aligned}
$$

Remark 3.20. In the special case $j(\nabla u)=|\nabla u|^{2}$ and $A^{0}=\alpha I$, one can check that $p^{0}=2 u^{0} / \alpha$ and the optimal direction of lamination is at each point perpendicular to $\nabla u^{0}$, giving then $h\left(\xi^{*}\right)=0$.

Remark 3.21. A similar treatment is possible for an objective function depending on the flux, namely

$$
J(\chi)=\int_{\Omega} j(A \nabla u) \mathrm{d} x .
$$

For details we refer to our preprint [2].

\subsection{Multiple loads}

We consider now a so-called multiple loads problem, i.e. several state equations are associated to a single objective function. Furthermore, we also consider the case of a multi-physics problem, i.e. the coefficients of the different state equations can be different although they share the same geometry or microstructure (a typical example would be thermo-elasticity where a conductivity problem is coupled to an elasticity system). For simplicity we restrict ourselves to two state equations in conductivity, but the same results would hold true for more equations. We consider an objective function that depends on two states $u$ and $v$

$$
J(\chi)=\int_{\Omega} j(u, v) \mathrm{d} x
$$

where $u$ is the solution of (3) with coefficients $A(x)=A^{0}(1+\eta \chi(x))$ and $v$ is the solution of

$$
\left.\begin{array}{r}
-\operatorname{div}(B \nabla v)=\tilde{f} \text { in } \Omega \\
v=0 \text { on } \Gamma_{D} \\
B \nabla v \cdot n=\tilde{g} \text { on } \Gamma_{N},
\end{array}\right\}
$$


with a different conductivity tensor

$$
B(x)=(1-\chi(x)) B^{0}+\chi(x) B^{1}=B^{0}(1+\eta \chi(x)) .
$$

As before we assume that the integrand $j$ is of class $C^{3}$ with adequate growth conditions with respect to its two arguments (the objective function could as well depend on a boundary integral without any conceptual additional difficulty). We denote by $j_{u}, j_{v}$ its partial first-order derivatives, and $j_{u u}, j_{v v}, j_{u v}$ its second-order derivatives.

Making a second-order asymptotic expansion with respect to $\eta$ we obtain

$$
\begin{aligned}
\mathcal{J}_{s a}\left(u^{i}, v^{j}\right)_{i, j=0,1,2}= & \int_{\Omega} j\left(u^{0}, v^{0}\right) \mathrm{d} x+\eta \int_{\Omega}\left(j_{u}\left(u^{0}, v^{0}\right) u^{1}+j_{v}\left(u^{0}, v^{0}\right) v^{1}\right) \mathrm{d} x \\
& +\eta^{2} \int_{\Omega}\left(j_{u}\left(u^{0}, v^{0}\right) u^{2}+j_{v}\left(u^{0}, v^{0}\right) v^{2}\right) \mathrm{d} x \\
& +\frac{1}{2} \eta^{2} \int_{\Omega}\left(j_{u u}\left(u^{0}, v^{0}\right)\left(u^{1}\right)^{2}+2 j_{u v}\left(u^{0}, v^{0}\right) u^{1} v^{1}+j_{v v}\left(u^{0}, v^{0}\right)\left(v^{1}\right)^{2}\right) \mathrm{d} x,
\end{aligned}
$$

where $u^{0}, u^{1}, u^{2}$ are solutions of (7), (8), and (9), respectively, while $v^{0}, v^{1}, v^{2}$ are solutions of similar equations involving $B^{0}$ instead of $A^{0}$ as tensor. The relaxation of the second-order small amplitude problem is easy to compute following the method described in the previous sections. The objective function becomes

$$
J_{s a}^{*}(\theta, \nu)=\mathcal{J}_{s a}\left(u^{i}, v^{j}\right)_{i, j=0,1,2}
$$

where $u^{0}, u^{1}, u^{2}$ are now solutions of the relaxed state equations (7), (12), (13), respectively, and $v^{0}, v^{1}, v^{2}$ solutions of the same relaxed equations where the tensor $A^{0}$ is replaced by $B^{0}$. Remark that there is a single $H$-measure $\nu$ since the same microstructure $\chi$ appears in both equations (3) and (39).

In order to show that $J_{s a}^{*}(\theta, \nu)$ is explicitly linear in $\nu$ we use two independent adjoints $p^{0}, q^{0}$, solutions of

$$
\begin{aligned}
& -\operatorname{div}\left(A^{0} \nabla p^{0}\right)=j_{u}\left(u^{0}, v^{0}\right) \text { in } \Omega \\
& -\operatorname{div}\left(B^{0} \nabla q^{0}\right)=j_{v}\left(u^{0}, v^{0}\right) \text { in } \Omega \\
& p^{0}=q^{0}=0 \text { on } \Gamma_{D}
\end{aligned}
$$

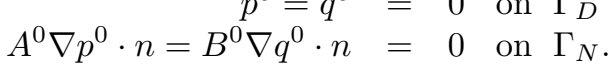

These adjoint states allow us to eliminate $u^{2}, v^{2}$ and to obtain

$$
\begin{aligned}
J_{s a}^{*}(\theta, \nu)= & \int_{\Omega} j\left(u^{0}, v^{0}\right) \mathrm{d} x+\eta \int_{\Omega}\left(j_{u}\left(u^{0}, v^{0}\right) u^{1}+j_{v}\left(u^{0}, v^{0}\right) v^{1}\right) \mathrm{d} x \\
& +\frac{1}{2} \eta^{2} \int_{\Omega}\left(j_{u u}\left(u^{0}, v^{0}\right)\left(u^{1}\right)^{2}+2 j_{u v}\left(u^{0}, v^{0}\right) u^{1} v^{1}+j_{v v}\left(u^{0}, v^{0}\right)\left(v^{1}\right)^{2}\right) \mathrm{d} x \\
& -\eta^{2} \int_{\Omega} \theta A^{0} \nabla u^{1} \cdot \nabla p^{0} \mathrm{~d} x-\eta^{2} \int_{\Omega} \theta B^{0} \nabla v^{1} \cdot \nabla q^{0} \mathrm{~d} x \\
& +\eta^{2} \int_{\Omega} \theta(1-\theta)\left(\int_{\mathbb{S}^{N-1}} h(\xi) \nu(x, \mathrm{~d} \xi)\right) \mathrm{d} x
\end{aligned}
$$

with

$$
h(\xi)=\frac{\xi \otimes \xi}{A^{0} \xi \cdot \xi} A^{0} \nabla u^{0} \cdot A^{0} \nabla p^{0}+\frac{\xi \otimes \xi}{B^{0} \xi \cdot \xi} B^{0} \nabla v^{0} \cdot B^{0} \nabla q^{0} .
$$


The minimization of $J_{s a}^{*}(\theta, \nu)$ with respect to $\nu$ can be achieved by a Dirac mass $\nu^{*}$ concentrated at a direction $\xi^{*}$ (independent of $\theta$ ) which minimizes the function $h(\xi)$. Therefore, once again, simple laminates are optimal microstructures for this multiple loads (or even multi-physics) problem.

\section{LINEARIZED ELASTICITY}

\subsection{Small amplitude asymptotic}

We generalize the results of the previous section to the system of linearized elasticity. We consider mixtures of two linear isotropic phases with elastic properties

$$
A^{0}=2 \mu I_{4}+\lambda I_{2} \otimes I_{2}, \quad A^{1}=2 \mu(1+\eta) I_{4}+\lambda(1+\eta) I_{2} \otimes I_{2},
$$

where $\eta$ is a small parameter, i.e. $|\eta| \ll 1$. For a characteristic function $\chi$ of the region occupied by phase $A^{1}$ we introduce the mixture elasticity tensor

$$
A(x)=A^{0}(1+\eta \chi(x)) .
$$

For a smooth bounded open set $\Omega \subset \mathbb{R}^{N}$, with boundary $\partial \Omega=\Gamma_{D} \cup \Gamma_{N}$, and for given loading forces $f \in L^{2}(\Omega)^{N}$ and $g \in L^{2}(\partial \Omega)^{N}$, we consider the following boundary value problem

$$
\left.\begin{array}{rlll}
-\operatorname{div}(A e(u)) & = & f & \text { in } \Omega \\
u & = & 0 & \text { on } \Gamma_{D} \\
A e(u) n & = & & \text { on } \Gamma_{N},
\end{array}\right\}
$$

where $e(u)=\frac{1}{2}\left(\nabla u+(\nabla u)^{T}\right)$ denotes the strain tensor. It is well known that (44) admits a unique solution in $H^{1}(\Omega)^{N}$. Typically we want to minimize an objective function depending on the strain tensor

$$
J(\chi)=\int_{\Omega} j(e(u)) \mathrm{d} x
$$

where the integrand $j$ is of class $C^{3}$ with quadratic growth at infinity. The case of an objective function depending on the stress tensor $\mathrm{Ae}(u)$ is completely similar and can be found in our preprint [2].

Assuming that the two phases have prescribed volume fractions, $\Theta$ for $A^{1}$ and $1-\Theta$ for $A^{0}$, with $\Theta \in(0,1)$, and recalling definition $(4)$ of the admissible set $\mathcal{U}_{a d}$, the large amplitude optimal design problem is

$$
\inf _{\chi \in \mathcal{U}_{a d}} J(\chi)
$$

We perform a second-order expansion with respect to $\eta$ in the state equation and in the objective function

$$
u=u^{0}+\eta u^{1}+\eta^{2} u^{2}+O\left(\eta^{3}\right) .
$$

Plugging this ansatz in (44) yields three equations for $\left(u^{0}, u^{1}, u^{2}\right)$

$$
\begin{aligned}
& \left.\begin{array}{rlll}
-\operatorname{div}\left(A^{0} e\left(u^{0}\right)\right) & = & \text { in } \Omega \\
u^{0} & =0 & \text { on } \Gamma_{D} \\
A^{0} e\left(u^{0}\right) n & =g & \text { on } \Gamma_{N},
\end{array}\right\} \\
& \left.\begin{array}{rl}
-\operatorname{div}\left(A^{0} e\left(u^{1}\right)\right) & =\operatorname{div}\left(\chi A^{0} e\left(u^{0}\right)\right) \text { in } \Omega \\
u^{1} & =0 \text { on } \Gamma_{D} \\
A^{0} e\left(u^{1}\right) n & =-\chi A^{0} e\left(u^{0}\right) n \text { on } \Gamma_{N},
\end{array}\right\}
\end{aligned}
$$




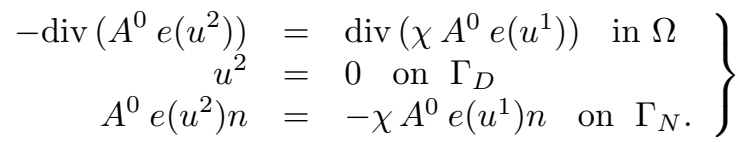

Similarly, we make a Taylor expansion in the objective function and, neglecting the remainder term, we obtain

$$
\begin{aligned}
J_{s a}(\chi)= & \int_{\Omega} j\left(e\left(u_{0}\right)\right) \mathrm{d} x+\eta \int_{\Omega} j^{\prime}\left(e\left(u_{0}\right)\right): e\left(u^{1}\right) \mathrm{d} x \\
& +\eta^{2} \int_{\Omega}\left(j^{\prime}\left(e\left(u_{0}\right)\right): e\left(u^{2}\right)+\frac{1}{2} j^{\prime \prime}\left(e\left(u_{0}\right)\right) e\left(u^{1}\right): e\left(u^{1}\right)\right) \mathrm{d} x .
\end{aligned}
$$

We thus obtain the equivalent of Definition 3.1 in the elasticity context.

Definition 4.1. We call "small amplitude" optimal design problem the second-order asymptotic of problem (45), namely

$$
\inf _{\chi \in \mathcal{U}_{a d}} J_{s a}(\chi)
$$

where $J_{s a}(\chi)$ is defined by (49), and $u^{0}, u^{1}, u^{2}$ are solutions of the state equations (46), (47), (48) respectively.

\subsection{Relaxation by $H$-measures}

As usual, (50) is ill-posed because it does not admit a minimizer in general. Therefore we relax it by using $H$-measures following the procedure in Section 3.2. Let $\chi_{n}$ be a sequence (minimizing or not) of characteristic functions which, up to a subsequence converges weakly-* to a limit density $\theta$ in $L^{\infty}(\Omega ;[0,1])$. We denote by $u^{0}, u_{n}^{1}, u_{n}^{2}$ the solutions of (46), (47), and (48) respectively, associated to $\chi_{n}$. Obviously we obtain that $u_{n}^{1}$ converges weakly to $u^{1}$ in $H^{1}(\Omega)^{N}$ which is the solution of

$$
\left.\begin{array}{rlr}
-\operatorname{div}\left(A^{0} e\left(u^{1}\right)\right) & =\operatorname{div}\left(\theta A^{0} e\left(u^{0}\right)\right) & \text { in } \Omega \\
u^{1} & =0 \text { on } \Gamma_{D} \\
A^{0} e\left(u^{1}\right) n & =-\theta A^{0} e\left(u^{0}\right) n \text { on } \Gamma_{N} .
\end{array}\right\}
$$

To pass to the limit in (48) we need first to compute the limit of $\chi_{n} e\left(u_{n}^{1}\right)$ by means of $H$-measures. From (47) we know that $e\left(u_{n}^{1}\right)$ depends linearly on $\chi_{n}$ through a pseudo-differential operator $q$, homogeneous of order 0 , the symbol of which is

$$
q(x, \xi)=-\frac{\sigma^{0} \xi \otimes \xi+\xi \otimes \sigma^{0} \xi}{2 \mu|\xi|^{2}}+\frac{(\mu+\lambda)\left(\sigma^{0} \xi \cdot \xi\right) \xi \otimes \xi}{\mu(2 \mu+\lambda)|\xi|^{4}} \quad \text { with } \sigma^{0}(x)=A^{0} e\left(u^{0}\right)(x) .
$$

The computation of this symbol is a classical result in the Hashin-Shtrikman variational principle (see e.g. the proof of Thm. 2.3.11 in [1]). Then, denoting by $\theta(1-\theta) \nu(\mathrm{d} x, \mathrm{~d} \xi)$ the $H$-measure of the sequence $\chi_{n}$, and applying Theorem 2.2, we deduce that

$$
\begin{aligned}
\lim _{n \rightarrow+\infty} \int_{\Omega} \chi_{n} A^{0} e\left(u_{n}^{1}\right) \cdot e(\phi) \mathrm{d} x & =\int_{\Omega} \theta A^{0} e\left(u^{1}\right) \cdot e(\phi) \mathrm{d} x \\
& -\int_{\Omega} \int_{\mathbb{S}_{N-1}} \theta(1-\theta) f_{A^{0}}(\xi) A^{0} e\left(u^{0}\right) \cdot A^{0} e(\phi) \nu(\mathrm{d} x, \mathrm{~d} \xi)
\end{aligned}
$$

where $f_{A^{0}}(\xi)$ is a fourth-order tensor defined, for any symmetric matrices $\sigma, \sigma^{\prime}$, by

$$
f_{A^{0}}(\xi) \sigma \cdot \sigma^{\prime}=\frac{\sigma \xi \cdot \sigma^{\prime} \xi}{\mu}-\frac{(\mu+\lambda)(\sigma \xi \cdot \xi)\left(\sigma^{\prime} \xi \cdot \xi\right)}{\mu(2 \mu+\lambda)} .
$$


Introducing a fourth-order tensor $M(x)$ defined, for any symmetric matrix $\sigma$, by

$$
M \sigma \cdot \sigma=\int_{\mathbb{S}^{N-1}} f_{A^{0}}(\xi) \sigma \cdot \sigma \nu(x, \mathrm{~d} \xi)
$$

we obtain that the weak limit in $H^{1}(\Omega)^{N}$ of $u_{n}^{2}$ is $u^{2}$, the unique solution in $H^{1}(\Omega)^{N}$ of

$$
\left.\begin{array}{rl}
-\operatorname{div}\left(A^{0} e\left(u^{2}\right)\right) & =\operatorname{div}\left(\theta A^{0} e\left(u^{1}\right)\right)-\operatorname{div}\left(\theta(1-\theta) A^{0} M A^{0} e\left(u^{0}\right)\right) \text { in } \Omega \\
u^{2} & =0 \text { on } \Gamma_{D} \\
A^{0} e\left(u^{2}\right) n & =-\theta A^{0} e\left(u^{1}\right) n+\theta(1-\theta) A^{0} M A^{0} e\left(u^{0}\right) n \text { on } \Gamma_{N} .
\end{array}\right\}
$$

To pass to the limit in (49) we use a computation similar to that in Section 3.5, which yields the relaxed objective function

$$
\begin{aligned}
J_{s a}^{*}(\theta, \nu) & =\int_{\Omega} j\left(e\left(u^{0}\right)\right) \mathrm{d} x-\eta \int_{\Omega} \theta A^{0} e\left(u^{0}\right): e\left(p^{0}\right) \mathrm{d} x-\eta^{2} \int_{\Omega} \theta A^{0} e\left(u^{1}\right): e\left(p^{0}\right) \mathrm{d} x \\
& +\frac{\eta^{2}}{2} \int_{\Omega} j^{\prime \prime}\left(e\left(u^{0}\right)\right) e\left(u^{1}\right): e\left(u^{1}\right) \mathrm{d} x+\eta^{2} \int_{\Omega} \theta(1-\theta) h_{2}(\xi) \nu(\mathrm{d} x, \mathrm{~d} \xi),
\end{aligned}
$$

where $u^{1}$ is the solution to $(51)$, and the first adjoint state $p^{0}$ is the solution in $H^{1}(\Omega)^{N}$ of

$$
\left.\begin{array}{rl}
-\operatorname{div}\left(A^{0} e\left(p^{0}\right)\right) & =-\operatorname{div} j^{\prime}\left(e\left(u^{0}\right)\right) \text { in } \Omega \\
p^{0} & =0 \text { on } \Gamma_{D} \\
\left(A^{0} e\left(p^{0}\right)\right) n & =j^{\prime}\left(e\left(u^{0}\right)\right) n \text { on } \Gamma_{N}
\end{array}\right\}
$$

and

$$
\begin{aligned}
h_{2}(\xi)= & \frac{1}{\mu} A^{0} e\left(u^{0}\right) \xi \cdot A^{0} e\left(p^{0}\right) \xi-\frac{\mu+\lambda}{\mu(2 \mu+\lambda)}\left(A^{0} e\left(u^{0}\right) \xi \cdot \xi\right)\left(A^{0} e\left(p^{0}\right) \xi \cdot \xi\right) \\
& +\frac{1}{2} j^{\prime \prime}\left(e\left(u^{0}\right)\right) q(x, \xi): q(x, \xi)
\end{aligned}
$$

where $q(x, \xi)$ is defined by $(52)$. Therefore we just have proved the following result.

Proposition 4.2. The relaxation of $(50)$ is thus

$$
\min _{(\theta, \nu) \in \mathcal{U}_{a d}^{*}} J_{s a}^{*}(\theta, \nu)
$$

where $\mathcal{U}_{\text {ad }}^{*}$ is defined by (16). More precisely,

1. there exists at least one minimizer $(\theta, \nu)$ of $(57)$;

2. any minimizer $(\theta, \nu)$ of $(57)$ is attained by a minimizing sequence $\chi_{n}$ of (50) in the sense that $\chi_{n}$ converges weakly- * to $\theta$ in $L^{\infty}(\Omega), \nu$ is the $H$-measure of $\left(\chi_{n}-\theta\right)$, and $\lim _{n \rightarrow+\infty} J_{s a}\left(\chi_{n}\right)=J_{s a}^{*}(\theta, \nu)$;

3. any minimizing sequence $\chi_{n}$ of (50) converges in the previous sense to a minimizer $(\theta, \nu)$ of $(57)$.

\subsection{Optimality conditions}

As in Section 3.4 we simplify the relaxed small amplitude problem (57) by using optimality conditions which show that optimal microstructures can always be found in the class of simple or rank-one laminates. We do not give all the details and the interested reader is referred to our preprint [2]. 
Proposition 4.3. The relaxed small amplitude problem (57) can be simplified by restricting the set of probability measures $\mathcal{P}\left(\Omega, \mathbb{S}_{N-1}\right)$, defined by $(2)$, to its subset of Dirac masses. More precisely, there exists an optimal design solution of

$$
\min _{(\theta, \nu) \in \mathcal{U}_{a d}^{s l}} J_{s a}^{*}(\theta, \nu)
$$

where $\mathcal{U}_{a d}^{s l} \subset \mathcal{U}_{a d}^{*}$ is defined by (26). Furthermore, the optimal Dirac mass $H$-measure in (58) does not depend on the density $\theta$.

Proof. Once again the key point is that $J_{s a}^{*}$ is linear in $\nu$. Thus, to minimize $J_{s a}^{*}$, it is enough to take $\nu$ as a Dirac mass in a direction $\xi^{*}$ which minimizes $h_{2}(\xi)$ in $\mathbb{S}^{N-1}$.

After elimination of $\nu$, the objective function $J_{s a}^{*}(\theta)$ is differentiable with respect to $\theta$ with directional derivative given by

$$
\begin{aligned}
\frac{\partial J_{s a}^{*}}{\partial \theta}(s) & =-\eta \int_{\Omega} s A^{0} e\left(u^{0}\right): e\left(p^{0}\right) \mathrm{d} x+\eta^{2} \int_{\Omega} s A^{0} e\left(u^{0}\right): e\left(p^{1}\right) \mathrm{d} x \\
& -\eta^{2} \int_{\Omega} s A^{0} e\left(u^{1}\right): e\left(p^{0}\right) \mathrm{d} x+\eta^{2} \int_{\Omega} s(1-2 \theta) h_{2}\left(\xi^{*}\right) \mathrm{d} x
\end{aligned}
$$

where $p^{1}$ is a second adjoint state, solution in $H^{1}(\Omega)^{N}$ of

$$
\left.\begin{array}{rl}
-\operatorname{div}\left(A^{0} e\left(p^{1}\right)\right) & =\operatorname{div}\left(j^{\prime \prime}\left(e\left(u^{0}\right)\right) e\left(u^{1}\right)\right)-\operatorname{div}\left(\theta A^{0} e\left(p^{0}\right)\right) \text { in } \Omega \\
p^{1} & =0 \text { on } \Gamma_{D} \\
\left(A^{0} e\left(p^{1}\right)\right) n & =\left(\theta A^{0} e\left(p^{0}\right)-j^{\prime \prime}\left(e\left(u^{0}\right)\right) e\left(u^{1}\right)\right) n \text { on } \Gamma_{N} .
\end{array}\right\}
$$

\section{Algorithm And NUmerical examples}

\subsection{The optimization algorithm}

We describe the optimization algorithm that we implemented to solve numerically the relaxed problems obtained in the previous sections. All the examples will be in dimension two. Recall that there are two design parameters: the lamination angle and the local proportion $\theta$. We have proved that the lamination direction of the optimal microstructure does not depend on $\theta$, and that it is explicitly given in terms of $u^{0}$ and $p^{0}$ which do not vary during the optimization process. Therefore, the optimal lamination angle is computed once and for all before we start a gradient-based steepest descend method for the local proportion $\theta$.

The resulting algorithm reads as follows:

- Initialization:

- Compute $u^{0}$ and $p^{0}$.

- Compute the optimal direction of lamination $\xi^{*}$.

- Set $\theta=\theta_{0}$ a constant.

- Compute $u^{1,0}$ and $p^{1,0}$.

- Evaluate $J_{s a}^{*}\left(\theta_{0}\right)$.

- Iterations: for $k \geq 1$ and $k$ less than a maximal number of iterations:

(i) Compute the gradient $\frac{\partial J_{s a}^{*}}{\partial \theta}\left(\theta_{k-1}\right)$ based on $u^{0}, p^{0}, u^{1, k-1}$ and $p^{1, k-1}$.

(ii) Update the local proportion with a step size $t_{k}>0$ by

$$
\theta_{k}=\min \left(1, \max \left(0, \tilde{\theta}_{k}\right)\right) \text { with } \tilde{\theta}_{k}=\theta_{k-1}-t_{k} \frac{\partial J_{s a}^{*}}{\partial \theta}\left(\theta_{k-1}\right)+\Lambda_{k}
$$

where $\Lambda_{k}$ is the Lagrange multiplier for the volume constraint.

(iii) Compute $u^{1, k}$ and evaluate $J_{s a}^{*}\left(\theta_{k}\right)$. 


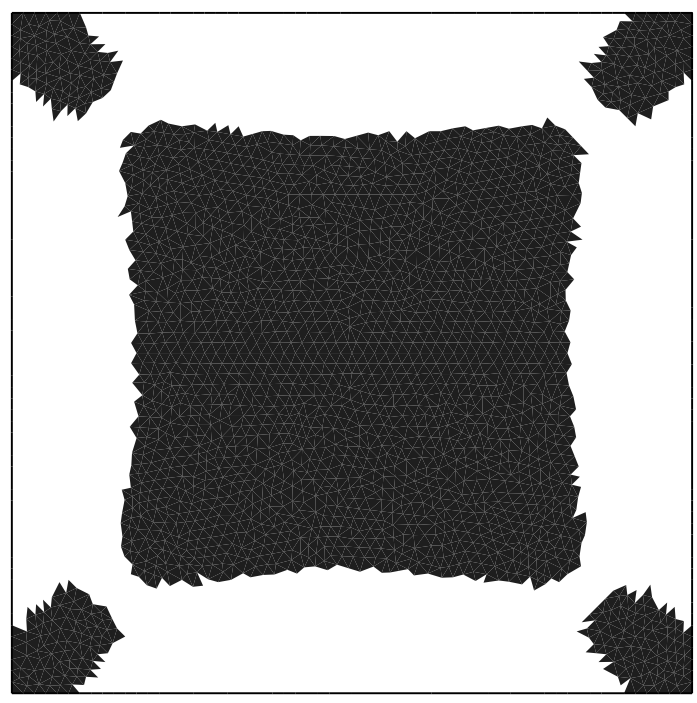

FIGURE 1. Compliance maximization: $\eta=-0.5$, volume $=50 \%$.

(iv) If $J_{s a}^{*}\left(\theta_{k}\right)<J_{s a}^{*}\left(\theta_{k-1}\right)$ : compute $p^{1, k}$ and make $k=k+1$. Go to step (i).

(v) If $J_{s a}^{*}\left(\theta_{k}\right) \geq J_{s a}^{*}\left(\theta_{k-1}\right)$ : reduce the step size $t_{k}$ and re-do steps (ii)-(v).

The volume constraint $\int_{\Omega} \theta_{k}(x) \mathrm{d} x=\Theta$ is enforced by adjusting the Lagrange multiplier $\Lambda_{k}$ by a simple dichotomy at each iteration.

The boundary value problems are solved using FreeFem $++[12]$ and we take advantage of the fact that all the problems we need to solve have the same elliptic differential operator, namely div $\left(A^{0} \nabla\right)$. Therefore the factorization of the stiffness matrix is performed only once during the initialization and is saved for all subsequent finite elements resolutions during the iterations. This of course speeds up considerably the code. The computational domain $\Omega$ is discretized by triangles. For all states $u^{i}$ and adjoint states $p^{i}$ we use $P_{2}$ Finite Elements, while the local proportion $\theta$ is discretized with $P_{0}$ Finite Elements (as well as the lamination direction $\xi^{*}$ ). As is well known (see $[1,6]$ and references therein) we prefer the $P_{2}-P_{0}$ combination to the simpler $P_{1}-P_{0}$ in order to avoid the so-called checkerboard numerical instability.

The subsequent figures show the local proportion of the material with higher conductivity or with higher stiffness, meaning higher values of both Lamé parameters. In other words, if $\eta$ is negative (which is always the case below), we display $(1-\theta)$. The volume, when mentioned in the caption, always refers to the percentage of volume occupied by the better conductor or the stiffer material. For some numerical examples we also show a plot of the values taken by the objective function during the optimization process.

\subsection{Diffusion problem}

Since the inception of the homogenization method a classical test case is the so-called torsion problem (see [1] for further references). It amounts to solve in the unit square $\Omega=(0,1) \times(0,1)$ the following problem

$$
\left.\begin{array}{r}
-\operatorname{div}(A \nabla u)=1 \quad \text { in } \Omega \\
u=0 \quad \text { in } \partial \Omega
\end{array}\right\}
$$

with $A(x)=(1-\chi(x)) A^{0}+\chi(x) A^{1}=A^{0}(1+\eta \chi(x))$, in order to minimize $J_{1}(\chi)=-\int_{\Omega} u \mathrm{~d} x$, i.e. to maximize the compliance. We solve the relaxed small amplitude version of this problem, as derived in Section 3.4: the resulting optimal design is displayed in Figure 1. Both phases have equal proportion and their conductivities are 0.5 and 1 , which yields a small amplitude $\eta=-0.5$. This figure can be compared to Figure 5.1 in [1] 


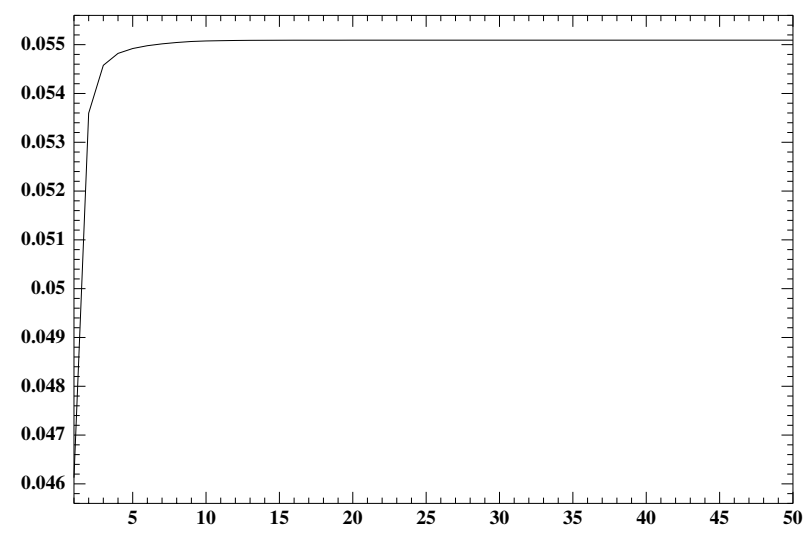

FiguRE 2. Convergence history of compliance maximization: $\eta=-0.5$, volume $=50 \%$.

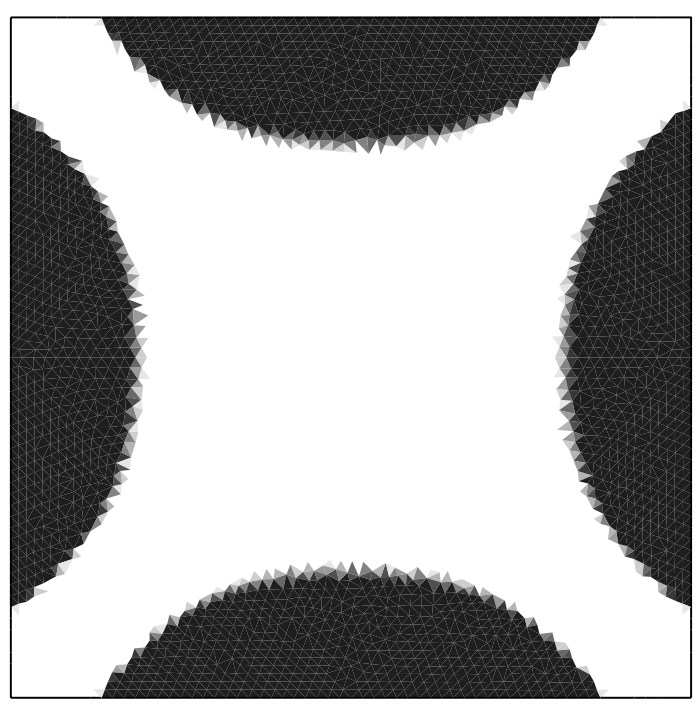

Figure 3. Gradient minimization. $\eta=-0.5$, volume $=40 \%$.

(up to the inversion of the gray scale), where the full homogenization involving the complete knowledge of the set $G_{\theta}$ was used: both optimal designs are very similar. Different values of $\eta$ (namely $\eta=-0.9$ or $\eta=-0.1$ ) yield also similar optimal designs. Furthermore, the obtained optimal design is convergent under mesh refinement.

We now turn to the minimization of $J_{2}(\chi)=\int_{\Omega}|\nabla u|^{2} \mathrm{~d} x$ for the same geometry and state equation. In Figure 3 we plot the resulting optimal shape for the relaxed small amplitude problem, as described in Section 3.5. The phase conductivities are 0.5 and 1 , and the proportion of the best conductor is $40 \%$. This resut is slightly different from that obtained by Lipton and Velo (see Fig. 1a in [17]; see also [8]) using a partial relaxation of the problem. Here also, different values of $\eta$ and different refinement of the mesh yield similar results.

In all examples of this section the square was discretized using about 8000 triangles. 


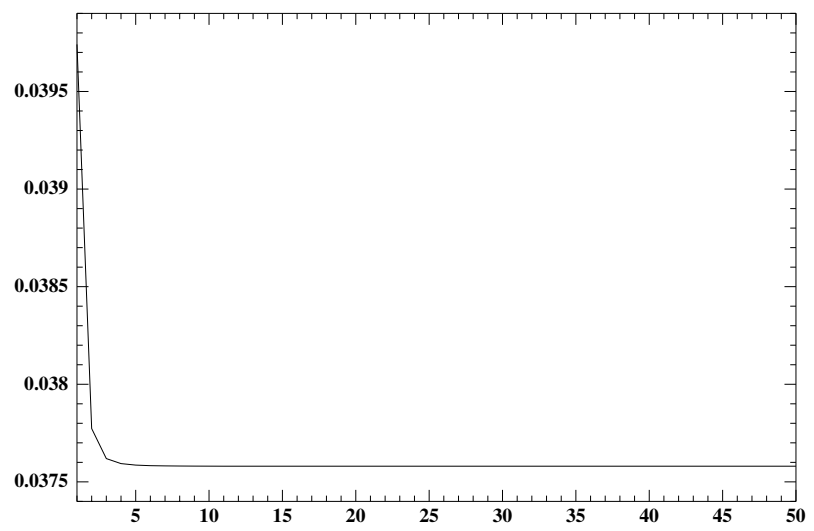

FIGURE 4. Convergence history of gradient minimization. $\eta=-0.5$, volume $=40 \%$.
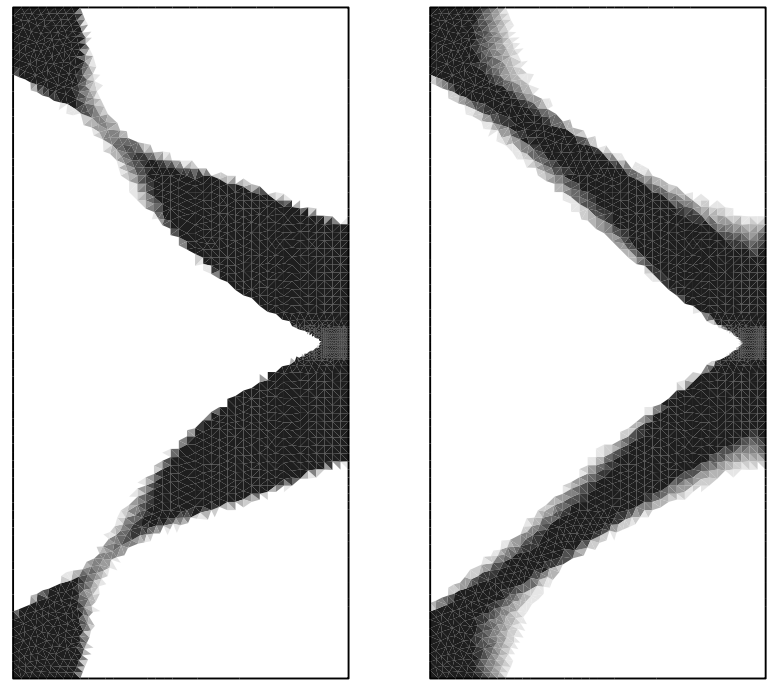

Figure 5. Compliance minimization for the short cantilever: $\eta=-0.1$ (left), $\eta=-0.99$ (right), volume $=25 \%$.

\subsection{Elasticity problem}

In all the following examples we take the reference material $A^{0}$ with Lamé coefficients $\lambda=0.73$ and $\mu=0.376$. As we said in the introduction, one should interpret the following results in the context of reinforcing a plane structure by adding to it a layer at a location that is optimal.

Let us first consider the so-called short cantilever problem subject to compliance minimization. We choose $\Omega=(0,1) \times(0,2)$ (discretized by 8765 triangles) and the state equation is given by

$$
\left.\begin{array}{r}
-\operatorname{div}(A e(u))=0 \quad \text { in } \Omega \\
u=0 \text { on } x=0 \\
(A e(u)) n=0 \text { on } \Gamma_{N 1} \\
(A e(u)) n=(0,-1)^{T} \quad \text { on } \Gamma_{N 2},
\end{array}\right\}
$$




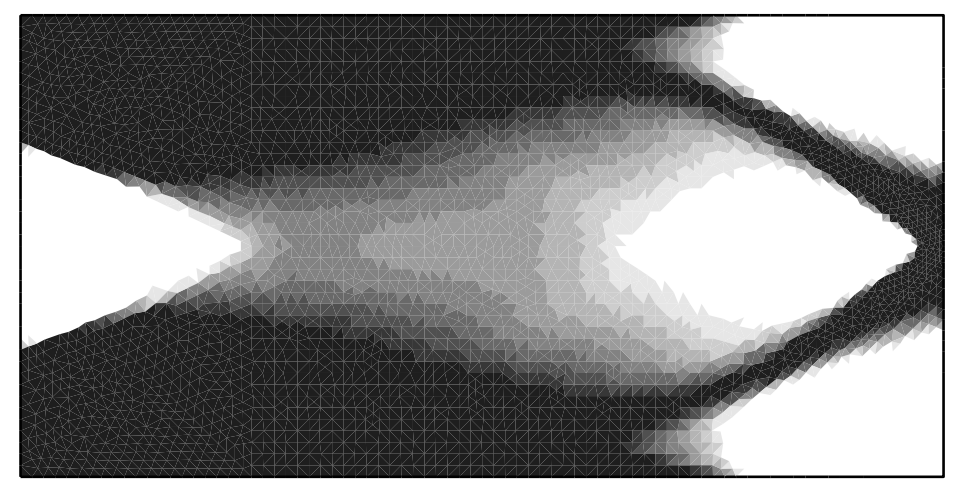

FiguRE 6. Compliance minimization of the long cantilever: $\eta=-0.99$, volume $=60 \%$.

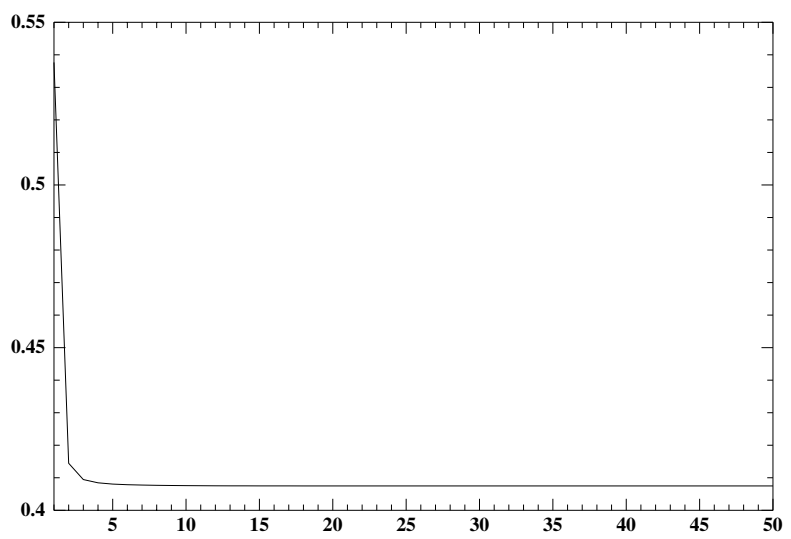

FiguRE 7. Convergence history for compliance minimization of the long cantilever: $\eta=-0.99$, volume $=60 \%$.

where $\Gamma_{N 1}=\{(x, y)$ s.t. $x \in(0,1)$ and $y \in\{0,2\}\} \cup\{(1, y)$ s.t. $y \in(0,0.95) \cup(1.05,2)\}$ and $\Gamma_{N 2}=\{(1, y)$ s.t. $y \in$ $(0.95,1.05)\}$.

After 50 iterations the resulting optimal designs for $\eta=-0.1$ and $\eta=-0.99$ are shown in Figure 5 (recall that dark colors correspond to the stiffer material). The latter design is quite similar to the usual short cantilever with two bars making a 90 degree angle at the position where the load is applied, giving then the impression that the approach developed here for the small amplitude case, might very well be used at least in some cases when the amplitude is not necessarily so small.

We now turn to the long cantilever problem, for which $\Omega=(0,2) \times(0,1)$ (discretized by 8088 triangles) with the same state equation where $\Gamma_{N 1}=\{(x, y)$ s.t. $x \in(0,2)$ and $y \in\{0,1\}\} \cup\{(2, y)$ s.t. $y \in(0,0.45) \cup(0.55,1)\}$ and $\Gamma_{N 2}=\{(2, y)$ s.t. $y \in(0.45,0.55)\}$. Figure 6 shows the optimal design for compliance minimization with $\eta=-0.99$. Remark in Figure 7 that the convergence is much quicker than the 50 iterations done. This design is different from the usual truss design obtained in the large amplitude case (see e.g. $[1,6]$ ), whatever the choice of the volume constraint (if it is too small, the two thin bars that link-up the two large concentrations of stiff material with the traction load, tend simply to disappear). In this case our small amplitude algorithm should not be used for standard structural optimization (where the amplitude is infinite). In Figure 8 we add to the compliance a term to penalize the presence of mixtures, in order to obtain a classical design starting from the optimal design of Figure 6 . 


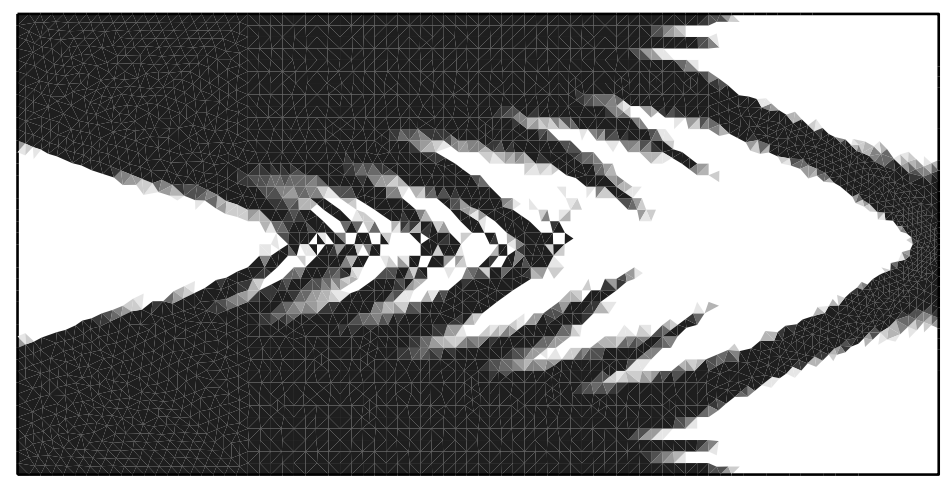

Figure 8. Compliance minimization with penalization for the long cantilever: $\eta=-0.99$, volume $=60 \%$.
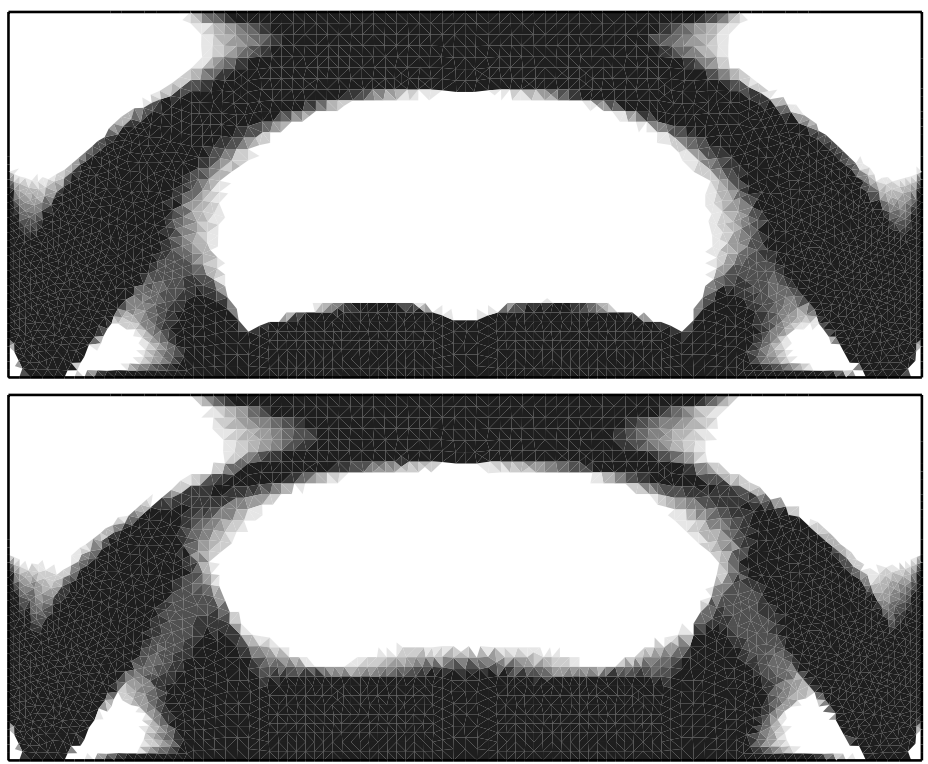

Figure 9. Compliance minimization of an arch with three loads: single load (top), multiple loads (bottom). $\eta=-0.99$, volume $=50 \%$.

The design of an arch is used to test the multiple loads case for compliance minimization presented in Section 3.6. The computational domain is $\Omega=(0,2) \times(0,0.8)$ (discretized by 7514 triangles). We first apply three forces together, i.e. we consider a single loading state equation

$$
\begin{aligned}
& -\operatorname{div}(A e(u))=0 \quad \text { in } \Omega \\
& u_{1}=u_{2}=0 \quad \text { on } x \in(0,0.1) \text { and } y=0 \\
& (A e(u)) n=(0,-1)^{T} \quad \text { on } \Gamma_{N 2},
\end{aligned}
$$

where $\Gamma_{N 1}=\{(x, 0)$ s.t. $x \in(0.1,0.45) \cup(0.55,0.95) \cup(1.05,1.45) \cup(1.55,1.9)\} \cup\{(x, y)$ s.t. $x \in\{0,2\}$ and $y \in$ $(0,0.8)\} \cup\{(x, 0.8)$ s.t. $x \in(0,2)\}$ and $\Gamma_{N 2}=\{(x, 0)$ s.t. $x \in(0.45,0.55) \cup(0.95,1.05) \cup(1.45,1.55)\}$. Second, we 


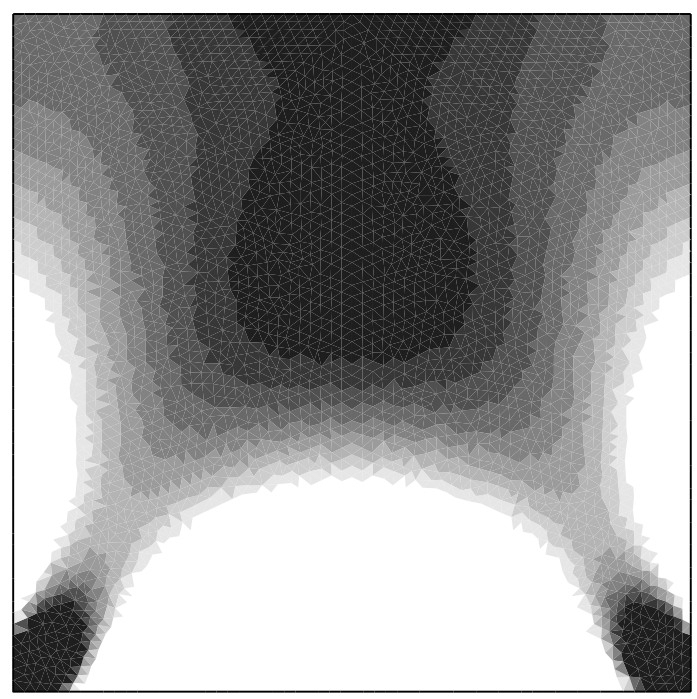

FIGURE 10. Strain minimization of a square clamped at the bottom and vertically loaded at the top: $\eta=-0.1$, volume $=50 \%$.

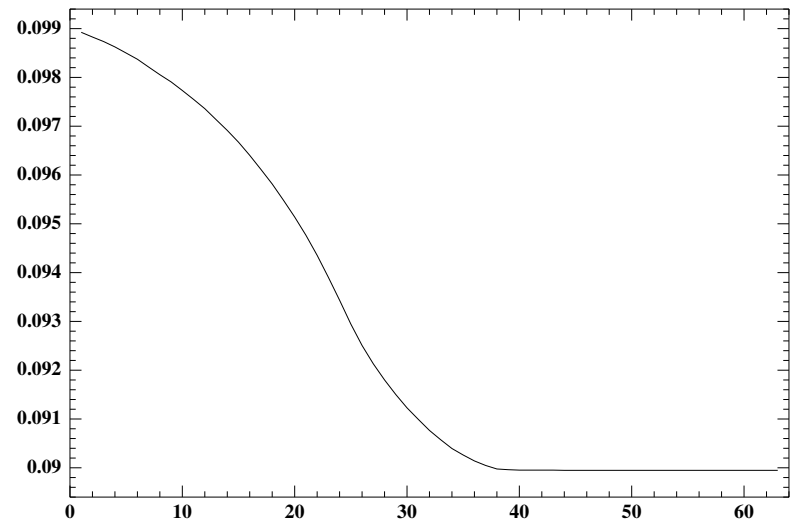

Figure 11. Convergence history of the strain minimization for the square: $\eta=-0.1$, volume $=50 \%$.

apply separately the three loads which yield three state equations, and we minimize the sum of the compliances. The corresponding optimal designs (after 50 iterations) are shown in Figure 9: they are similar, on the contrary of the large amplitude case in which the two optimal designs are different (see [1]).

Next we minimize the norm of the strain tensor, localized on a subdomain $\omega$, i.e. $J(\chi)=\int_{\omega}|e(u)|^{2} \mathrm{~d} x$, as presented in Section 4.3. The domain is the unit square $\Omega=(0,1)^{2}$, which is discretized with 8654 triangles, the subdomain is just $\omega=\Omega$, and the state equation is

$$
\begin{aligned}
& -\operatorname{div}(A e(u))=0 \text { in } \Omega \\
& u=0 \text { on } \Gamma_{D} \\
& (A e(u)) n=(0,0)^{T} \text { on } \Gamma_{N 1} \\
& (A e(u)) n=(0,-1)^{T} \text { on } \Gamma_{N 2} \text {, }
\end{aligned}
$$



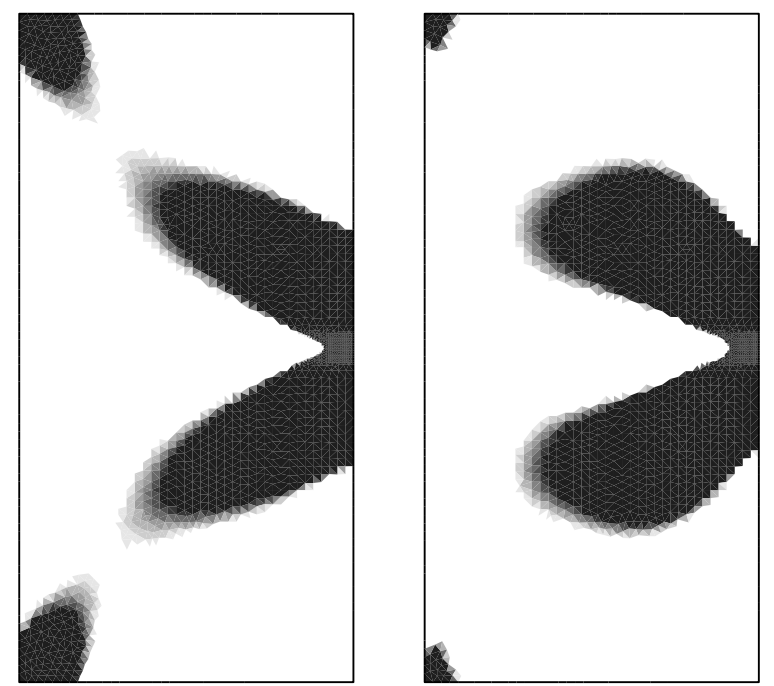

FiguRE 12. Strain minimizaton for the short cantilever: over the whole domain (left), or on a subdomain (right). $\eta=-0.1$, volume $=25 \%$.
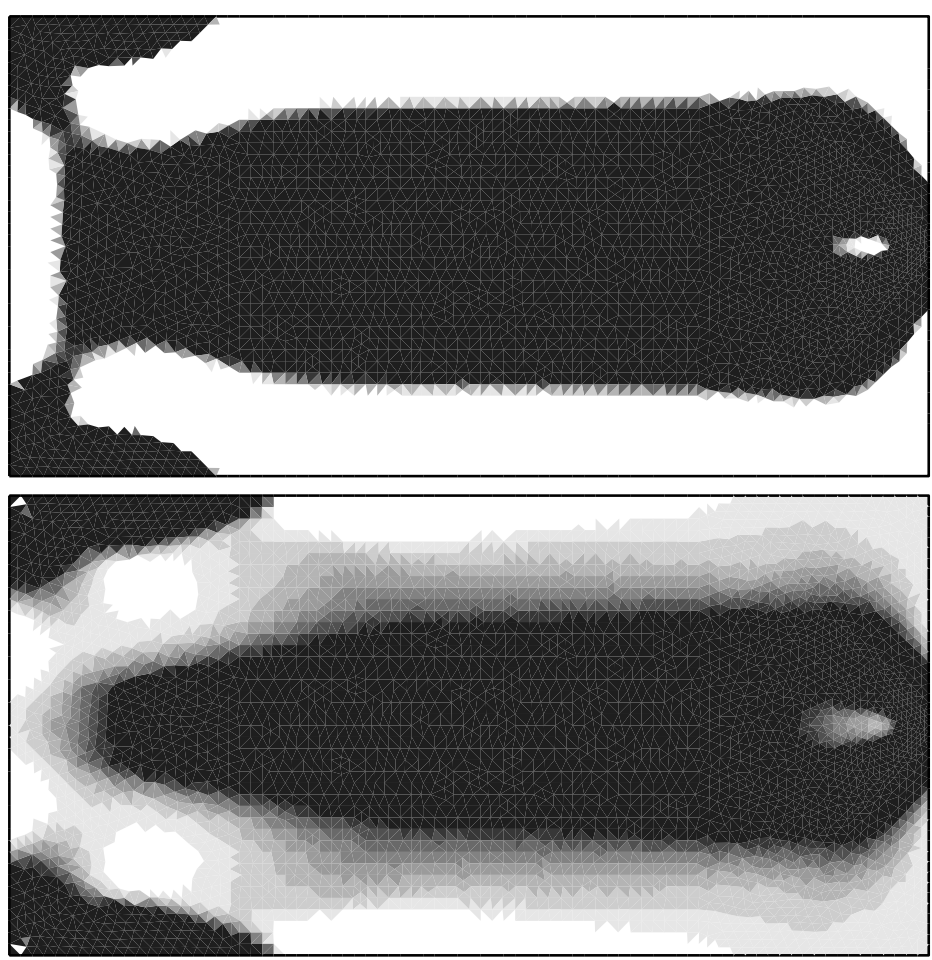

Figure 13. Strain minimization for the long cantilever on a subdomain. Volume $=60 \%$, $\eta=-0.1$ (above), $\eta=-0.99$ (below). 


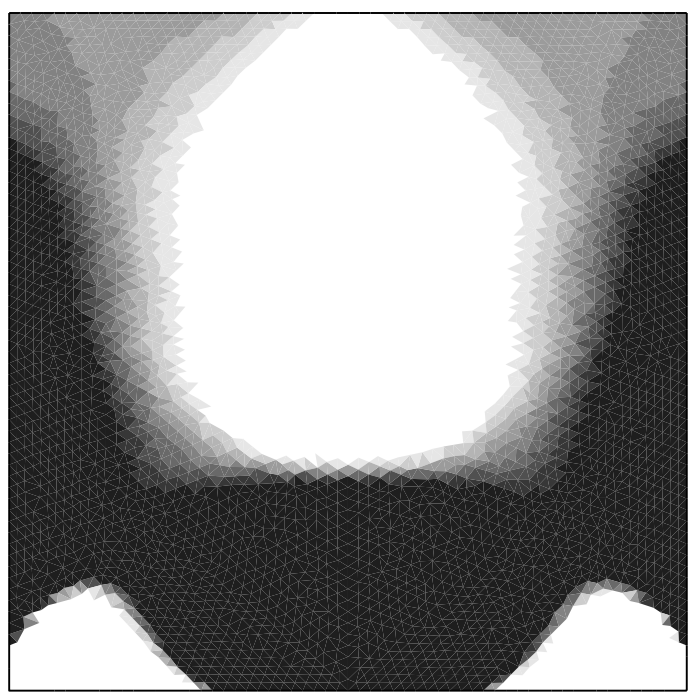

Figure 14. Stress minimization for elasticity, Dirichlet data homogeneous on bottom, Neumann homogeneous on the sides and vertical load on the top. $\eta=-0.1$, volume $=50 \%$.

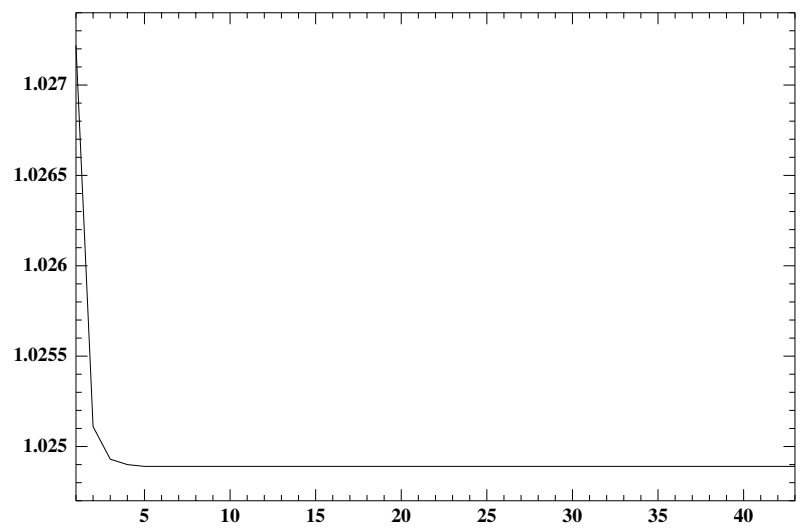

FigurE 15. Convergence history stress minimization for elasticity, Dirichlet data homogeneous on bottom, Neumann homogeneous on the sides and vertical load on the top. $\eta=-0.1$, volume $=50 \%$.

where $\Gamma_{D}=\left\{(x, 0) \in \mathbb{R}^{2}\right.$ s.t. $\left.x \in(0,1)\right\}, \Gamma_{N 1}=\left\{(x, y) \in \mathbb{R}^{2}\right.$ s.t. $x \in\{0,1\}$ and $\left.y \in(0,1)\right\}$ and $\Gamma_{N 2}=\{(x, 1) \in$ $\mathbb{R}^{2}$ s.t. $\left.x \in(0,1)\right\}$. The resulting optimal design, shown in Figure 10, looks like a bridge with two pillars. The convergence is smooth as can be checked in Figure 11.

We perform the same strain norm minimization for the short cantilever with either $\omega=\Omega$, or a smaller subset $\omega=(0.9,1.0) \times(0.9,1.1)$ around the loading force, see Figure 12 . The same minimization is done for the long cantilever with $\omega=(1.8,2.0) \times(0.4,0.6)$ and two different values of the amplitude parameter $\eta$, see Figure 13 .

Finally we minimize the norm of the stress, localized on a subdomain $\omega$, i.e. $J(\chi)=\int_{\omega}|A e(u)|^{2} \mathrm{~d} x$. First we consider the same square domain, loading state and subset $\omega=\Omega$ as already considered for the strain minimization in Figure 10. The resulting optimal design shown in Figure 14 is totally different when we minimize the stress, which, at least to us, is somewhat surprising. 

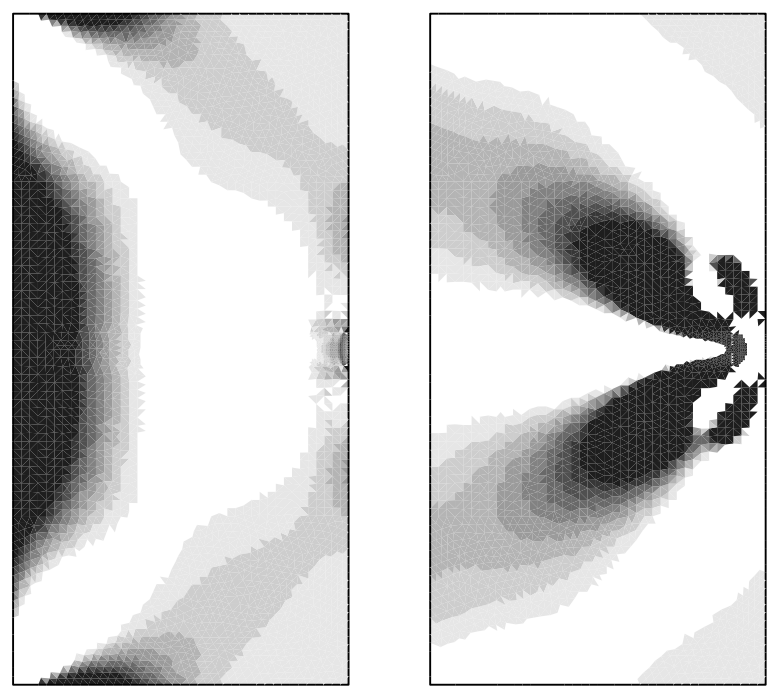

FiguRE 16. Stress minimization for the short cantilever: over the whole domain (left), or on a subdomain (right). $\eta=-0.1$, volume $=25 \%$.

Secondly we consider the short cantilever $\Omega=(0,1) \times(0,2)$ (discretized by 8765 triangles) with $\eta=-0.1$ and the same loading state as before. We compare the minimization over the whole domain with the one over a small box $\omega=(0.9 ; 1.0) \times(0.9 ; 1.1)$ close to the loading force on the right. Here again the optimal designs of Figure 16 are very different from those of Figures 5 and 12.

Acknowledgements. This work has partially been supported by the ECOS project C04E07 of cooperation between Chile and France, and by the ACI project GUIDOPT of the French Ministry of Research.

\section{REFERENCES}

[1] G. Allaire, Shape Optimization by the Homogenization Method. Springer-Verlag (2002).

[2] G. Allaire and S. Gutiérrez, Optimal design in small amplitude homogenization (extended version). Preprint available at http://www.cmap.polytechnique.fr/preprint/repository/576.pdf (2005).

[3] G. Allaire and F. Jouve, Optimal design of micro-mechanisms by the homogenization method. Eur. J. Finite Elements 11 (2002) 405-416.

[4] G. Allaire, F. Jouve and H. Maillot, Topology optimization for minimum stress design with the homogenization method. Struct. Multidiscip. Optim. 28 (2004) 87-98.

[5] J.C. Bellido and P. Pedregal, Explicit quasiconvexification for some cost functionals depending on derivatives of the state in optimal designing. Discr. Contin. Dyn. Syst. 8 (2002) 967-982.

[6] M.P. Bendsøe and O. Sigmund, Topology Optimization. Theory, Methods, and Applications. Springer-Verlag, New York (2003).

[7] A. Cherkaev, Variational Methods for Structural Optimization. Springer Verlag, New York (2000).

[8] A. Donoso and P. Pedregal, Optimal design of 2D conducting graded materials by minimizing quadratic functionals in the field. Struct. Multidiscip. Optim. 30 (2005) 360-367.

[9] P. Duysinx and M.P. Bendsøe, Topology optimization of continuum structures with local stress constraints. Int. J. Num. Meth. Engng. 43 (1998) 1453-1478.

[10] P. Gérard, Microlocal defect measures. Comm. Partial Diff. Equations 16 (1991) 1761-1794.

[11] Y. Grabovsky, Optimal design problems for two-phase conducting composites with weakly discontinuous objective functionals. Adv. Appl. Math. 27 (2001) 683-704.

[12] F. Hecht, O. Pironneau and K. Ohtsuka, FreeFem++ Manual. Downloadable at http://www.freefem.org

[13] L. Hörmander, The analysis of linear partial differential operators III. Springer, Berlin (1985).

[14] R.V. Kohn, Relaxation of a double-well energy. Cont. Mech. Thermodyn. 3 (1991) 193-236.

[15] R. Lipton, Relaxation through homogenization for optimal design problems with gradient constraints. J. Optim. Theory Appl. 114 (2002) 27-53. 
[16] R. Lipton, Stress constrained $G$ closure and relaxation of structural design problems. Quart. Appl. Math. 62 (2004) $295-321$.

[17] R. Lipton and A. Velo, Optimal design of gradient fields with applications to electrostatics. Nonlinear partial differential equations and their applications. Collège de France Seminar, Vol. XIV, Stud. Math. Appl. 31 (2002) 509-532.

[18] G. Milton, The theory of composites. Cambridge University Press (2001).

[19] F. Murat and L. Tartar, Calcul des Variations et Homogénéisation, Les Méthodes de l'Homogénéisation Théorie et Applications en Physique, Coll. Dir. Études et Recherches EDF, 57, Eyrolles, Paris (1985) 319-369. English translation in Topics in the mathematical modelling of composite materials, A. Cherkaev and R. Kohn Eds., Progress in Nonlinear Differential Equations and their Applications 31, Birkhäuser, Boston (1997).

[20] U. Raitums, The extension of extremal problems connected with a linear elliptic equation. Soviet Math. 19 (1978) $1342-1345$.

[21] L. Tartar, H-measures, a new approach for studying homogenization, oscillations and concentration effects in partial differential equations. Proc. Royal Soc. Edinburgh 115A (1990) 93-230.

[22] L. Tartar, Remarks on optimal design problems. Calculus of variations, homogenization and continuum mechanics (Marseille, 1993), World Sci. Publishing, River Edge, NJ, Ser. Adv. Math. Appl. Sci. 18 (1994) 279-296.

[23] L. Tartar, An introduction to the homogenization method in optimal design, in Optimal shape design (Tróia, 1998), A. Cellina and A. Ornelas Eds., Springer, Berlin, Lect. Notes Math. 1740 (2000) 47-156. 